\title{
FRAMED HOLOMORPHIC BUNDLES ON RATIONAL SURFACES
}

\author{
JOÃO PAULO SANTOS
}

\begin{abstract}
We study the moduli space of framed holomorphic bundles of any rank, over the blow-up of $\mathbb{C P}^{2}$ at $q$ points. For $c_{2}=1,2$ we introduce an open cover of the moduli space and describe its nerve. In the limit when $r \rightarrow \infty$ we use this result to obtain the homotopy type of the moduli spaces. In particular, we compute the cohomology of the moduli spaces.
\end{abstract}

\section{Introduction}

Fix a line $L_{\infty} \subset \mathbb{C P}^{2}$ and let $X_{q}$ denote the blow-up of $\mathbb{C P}^{2}$ at $q$ points $x_{1}, \ldots, x_{q} \notin L_{\infty}$. In this paper we will study the moduli space $\mathfrak{M}_{k}^{r}\left(X_{q}\right)$ of equivalence classes of pairs $(\mathcal{E}, \phi)$, where $\mathcal{E}$ is a holomorphic rank $r$ bundle over $X_{q}$ with $c_{1}=0$ and $c_{2}=k$, holomorphically trivial at $L_{\infty}$, and $\phi:\left.\mathcal{E}\right|_{L_{\infty}} \rightarrow \mathcal{O}_{L_{\infty}}^{r}$ is a holomorphic trivialization. This moduli space is a special case of the moduli of framed sheaves introduced in [15], [11]. See also [16]. The interest on these spaces was motivated by the study of moduli spaces of instantons: in [5], [17] it was shown that $\mathfrak{M}_{k}^{r}\left(X_{q}\right)$ is isomorphic as a real analytic space to the moduli space of based charge $k S U(r)$ instantons over a connected sum of $q$ copies of $\mathbb{C P}^{2}$. Monad descriptions for these spaces were introduced in [1], [7], for $X_{0}=\mathbb{C P}^{2}$, [4], [12] for $X_{1}$ and [14], [6], for the general case. In this paper we study the moduli spaces using results about bundles on the blow-up of a complex surface (see [19]): analyzing the effect of blowing up on the topology of the moduli space we reduce the study of the moduli spaces over $X_{q}$ to the moduli spaces over $X_{0}, X_{1}$. Our first result is

Theorem 1.1. Let $I \subset\{1, \ldots, q\}$ and write $|I|=\# I$. Then, for each $I$ with $|I| \leq k$ there is an open set $A_{I} \subset \mathfrak{M}_{k}^{r}\left(X_{q}\right)$ such that

(1) $\left\{A_{I}\right\}_{|I|=k}$ is an open cover of $\mathfrak{M}_{k}^{r}\left(X_{q}\right)$;

(2) $A_{I} \cap A_{J}=A_{I \cap J}$

(3) There are homeomorphisms $A_{I} \cong \mathfrak{M}_{|I|}^{r}\left(X_{q}\right)$.

For $k=1$ this describes $\mathfrak{M}_{1}^{r}\left(X_{q}\right)$ in terms of the moduli spaces over $X_{0}$ and $X_{1}$, which are well understood. Hence we can get a cell structure for $\mathfrak{M}_{1}^{r}\left(X_{q}\right)$. For $k=2$ we will prove:

Theorem 1.2. There are open sets $A_{i}, N_{i j} \subset \mathfrak{M}_{2}^{r}\left(X_{q}\right)$ such that

(1) $\left\{A_{i}\right\}_{i} \cup\left\{N_{i j}\right\}_{i<j}$ is an open cover of $\mathfrak{M}_{2}^{r}\left(X_{q}\right)$;

(2) There is an open set $A_{\emptyset} \subset \mathfrak{M}_{2}^{r}\left(X_{q}\right)$ such that $A_{i} \cap A_{j}=A_{\emptyset}$ for $i \neq j$.

(3) For $k \notin\{i, j\}, N_{i j} \cap A_{k}=N_{i j} \cap A_{\emptyset}$. For different sets $\{i, j\} \neq\{k, l\}$, $N_{i j} \cap N_{k l}=\emptyset$. 
(4) There are homotopy equivalences

$$
\begin{array}{ll}
A_{i} \simeq \mathfrak{M}_{2}^{r}\left(X_{1}\right) & A_{\emptyset} \simeq \mathfrak{M}_{2}^{r}\left(X_{0}\right) \\
N_{i j} \simeq \mathfrak{M}_{1}^{r}\left(X_{1}\right) \times \mathfrak{M}_{1}^{r}\left(X_{1}\right) & \\
N_{i j} \cap A_{i} \simeq \mathfrak{M}_{1}^{r}\left(X_{1}\right) \times \mathfrak{M}_{1}^{r}\left(X_{0}\right) & N_{i j} \cap A_{\emptyset} \simeq \mathfrak{M}_{1}^{r}\left(X_{0}\right) \times \mathfrak{M}_{1}^{r}\left(X_{0}\right)
\end{array}
$$

We then apply these results to the study of the rank stable moduli space, which is defined as follows: when $r_{2}>r_{1}$, there is a map $\mathfrak{M}_{k}^{r_{1}}\left(X_{q}\right) \rightarrow \mathfrak{M}_{k}^{r_{2}}\left(X_{q}\right)$ induced by taking direct sum with a trivial rank $r_{2}-r_{1}$ bundle. We define the rank stable moduli space as the direct limit $\mathfrak{M}_{k}^{\infty}\left(X_{q}\right) \stackrel{\text { def }}{=} \underset{r}{\lim } \mathfrak{M}_{k}^{r}\left(X_{q}\right)$. Using the monad descriptions, it was shown in [13], [18] and [2] that

$$
\mathfrak{M}_{k}^{\infty}\left(X_{0}\right) \simeq B U(k), \mathfrak{M}_{k}^{\infty}\left(X_{1}\right) \simeq B U(k) \times B U(k)
$$

We will prove as a corollary of theorem 1.1 that

Theorem 1.3. There is a homotopy equivalence

$$
\mathfrak{M}_{1}^{\infty}\left(X_{q}\right) \simeq B U(1) \times\left(\bigvee_{i=1}^{q} B U(1)\right)
$$

Together with the results of [5] and [17], this shows that for a large class of metrics conjecture 1.1 in [2] is false.

Using theorem 1.2 we will compute the cohomology of $\mathfrak{M}_{2}^{\infty}\left(X_{q}\right)$ :

Theorem 1.4. Let $K_{C} \subset \mathbb{Z}\left[x_{1}, x_{2}, x_{3}, x_{4}\right] \cong H^{*}\left(B U(1)^{\times 4}\right)$ be the ideal generated by the product $x_{1} x_{2}$ and let $K_{A} \subset \mathbb{Z}\left[a_{1}, k_{1}, a_{2}, k_{2}\right] \simeq H^{*}\left(B U(2)^{\times 2}\right)$ be the ideal generated by $k_{1}, k_{2}$. Then, as graded modules over $\mathbb{Z}$, we have an isomorphism

$$
H^{*}\left(\mathfrak{M}_{2}^{\infty}\left(X_{q}\right)\right) \cong \mathbb{Z}\left[a_{1}, a_{2}\right] \oplus K_{A}^{\oplus q} \oplus K_{C}^{\oplus \frac{q(q-1)}{2}}
$$

The plan of this paper is as follows: In section 2 we prove theorem 1.1 and in section 3 we use it to prove theorem 1.3. In section 4 we prove theorem 1.2 and in the next two sections we apply it to prove theorem 1.4: in section 5 we study the open cover from theorem 1.2 in the limit when $r \rightarrow \infty$; in section 6 we use the spectral sequence associated with this open cover (see [21]) to prove theorem 1.4. In the appendix we gather some results about the monad constructions of the moduli spaces for $q=0,1$.

This paper is based on results in the author's thesis [20].

\section{AN OPEN COVER OF $\mathfrak{M}_{k}^{r}\left(X_{q}\right)$}

Fix a subset $I \subset\{1, \ldots, q\}$ and let $\pi_{I}: X_{q} \rightarrow X_{|I|}(|I|=\# I)$ be the blow up at points $x_{j}, j \notin I . \pi_{I}$ induces a map

$$
\pi_{I}^{*}: \mathfrak{M}_{k}^{r}\left(X_{|I|}\right) \rightarrow \mathfrak{M}_{k}^{r}\left(X_{q}\right)
$$

Let $q \geq k$. The objective of this section is to prove

Theorem 2.1. $\left\{\pi_{I}^{*} \mathfrak{M}_{k}^{r}\left(X_{|I|}\right)\right\}_{|I|=k}$ is an open cover of $\mathfrak{M}_{k}^{r}\left(X_{q}\right)$. Furthermore

$$
\pi_{I}^{*} \mathfrak{M}_{k}^{r}\left(X_{|I|}\right) \cap \pi_{J}^{*} \mathfrak{M}_{k}^{r}\left(X_{|J|}\right)=\pi_{I \cap J}^{*} \mathfrak{M}_{k}^{r}\left(X_{|I \cap J|}\right)
$$


and we have isomorphisms

$$
\mathfrak{M}_{k}^{r}\left(X_{|I|}\right) \underset{\pi_{I *}}{\stackrel{\pi_{I}^{*}}{\cong}} \pi_{I}^{*} \mathfrak{M}_{k}^{r}\left(X_{|I|}\right)
$$

From this open cover we can build a spectral sequence converging to $H^{*}\left(\mathfrak{M}_{k}^{r}\left(X_{q}\right)\right)$. The case $k=2$ will be treated in section 7 . For the general case see [20], section 4.3. We turn now to the proof of theorem 2.1. We begin by proving the last statement:

Proposition 2.2. We have isomorphisms

$$
\mathfrak{M}_{k}^{r}\left(X_{|I|}\right) \underset{\pi_{I *}}{\stackrel{\pi_{I}^{*}}{\cong}} \pi_{I}^{*} \mathfrak{M}_{k}^{r}\left(X_{|I|}\right)
$$

where $\pi_{I}^{*}$ and $\pi_{I *}$ are inverses of each other. We also have

$$
\pi_{I}^{*} \mathfrak{M}_{k}^{r}\left(X_{|I|}\right)=\left\{\mathcal{E} \in \mathfrak{M}_{k}^{r}\left(X_{q}\right)|\mathcal{E}|_{L_{i}} \text { is trivial for } i \notin I\right\}
$$

Proof. From theorem 3.2 in [9] it follows that, if a bundle is trivial on the exceptional divisor then it is also trivial on a neighborhood of the exceptional divisor. Hence, a bundle $\mathcal{E} \rightarrow \tilde{X}$ on a blow up $\pi: \tilde{X} \rightarrow X$ is trivial on the exceptional divisor if and only if $\tilde{\mathcal{E}}=\pi^{*} \pi_{*} \tilde{\mathcal{E}}$. The statement of the proposition follows.

Proof of theorem 2.1. From proposition 2.2 it follows that

$$
\pi_{I}^{*} \mathfrak{M}_{k}^{r}\left(X_{|I|}\right) \cap \pi_{J}^{*} \mathfrak{M}_{k}^{r}\left(X_{|J|}\right)=\pi_{I \cap J}^{*} \mathfrak{M}_{k}^{r}\left(X_{|I \cap J|}\right)
$$

To show that $\mathfrak{M}_{k}^{r}\left(X_{q}\right) \subset \bigcup_{|I|=k} \pi_{I}^{*} \mathfrak{M}_{k}^{r}\left(X_{k}\right)$ we only need to show that a bundle $\mathcal{E} \in \mathfrak{M}_{k}^{r}\left(X_{q}\right)$ is trivial in at least $q-k$ exceptional lines $(q>k)$. We prove this result by induction in $q$. Assume $\mathcal{E}$ is not trivial in $L_{1}$. Let $p: X_{q} \rightarrow X_{q-1}$ be the blow up at $x_{1}$ and let $\mathcal{E}^{\prime}=\left(\pi_{*} \mathcal{E}\right)^{\vee \vee}$. Then $c_{2}\left(\mathcal{E}^{\prime}\right)<k$ so we can apply induction. The proof is completed by noting that we cannot have bundles with negative $c_{2}$ by Bogomolov inequality for framed bundles (see [15]).

Finally we have to show that $\pi_{I}^{*} \mathfrak{M}_{k}^{r}\left(X_{|I|}\right)$ is open. Let $H$ be an ample divisor. Choose $N$ such that $H^{i}(\mathcal{E}(N H))=0$ for all $\mathcal{E} \in \mathfrak{M}_{k}^{r}(\tilde{X})$. Then choose $M$ such that $\pi_{*} \mathcal{E}(N H+M L)$ is locally free. Consider the function $h^{1}: \mathfrak{M}_{k}^{r}(\tilde{X}) \rightarrow \mathbb{Z}$ defined by $h^{1}=\operatorname{dim} H^{1}(\mathcal{E}(N H+M L))$. Then, from the exact sequence

$$
0 \rightarrow \mathcal{E}(N H) \rightarrow \mathcal{E}(N H+M L) \rightarrow \mathcal{T} \rightarrow 0
$$

$(\mathcal{T}$ has support contained in $L)$ we get $H^{2}(\mathcal{E}(N H+M L))=H^{2}(\mathcal{T})=0$. Now notice that

$$
H^{0}(\mathcal{E}(N H+M L)) \cong H^{0}\left(\pi_{*} \mathcal{E}(N H+M L)\right)
$$

and, since by assumption $\pi_{*} \mathcal{E}(N H+M L)$ is locally free and $\pi_{*} H$ is ample, for $N$ large enough we get

$$
H^{i}\left(\pi_{*} \mathcal{E}(N H+M L)\right)=0 \text { for } i>0
$$

Hence, we get that

$$
h^{1}=\chi\left(\pi_{*} \mathcal{E}(N H+M L)\right)-\chi(\mathcal{E}(N H+M L))
$$

From Riemann-Roch theorem it follows that

$$
h^{1}=c_{2}(\mathcal{E})-c_{2}\left(\pi_{*} \mathcal{E}^{\vee \vee}\right)+f\left(N, M, c_{1}(X)\right)
$$

where $f$ does not depend on $\mathcal{E}$. The result then follows from the upper-semicontinuity of $h^{1}$ (see [10], chapter III, theorem 12.8). 


\section{The Charge One MOdUli SPACE}

The objective of this section is to prove theorem 1.3:

Theorem 3.1. There is a homotopy equivalence

$$
\mathfrak{M}_{1}^{\infty}\left(X_{q}\right) \simeq B U(1) \times\left(\bigvee_{i=1}^{q} B U(1)\right)
$$

From theorem 2.1 we know that

$$
\mathfrak{M}_{1}^{r}\left(X_{q}\right)=\bigcup_{l=1}^{q} \pi_{l}^{*} \mathfrak{M}_{1}^{r}\left(X_{1}\right), \pi_{i}^{*} \mathfrak{M}_{1}^{r}\left(X_{1}\right) \cap \pi_{j}^{*} \mathfrak{M}_{1}^{r}\left(X_{1}\right)=\pi_{\emptyset}^{*} \mathfrak{M}_{1}^{r}\left(X_{0}\right)(i \neq j)
$$

We begin by studying the maps $\pi_{\emptyset}^{*} \mathfrak{M}_{1}^{r}\left(X_{0}\right) \rightarrow \pi_{i}^{*} \mathfrak{M}_{1}^{r}\left(X_{1}\right)$.

Lemma 3.2. Let $\iota_{1}: \mathbb{C P}^{r} \rightarrow \mathbb{C P}^{r} \times \mathbb{C P}^{r}$ be the inclusion into the first factor: $\iota_{1}([u])=([u], *)$, where $*$ denotes the base point. Then there are homotopy equivalences $h_{0}: \mathbb{C P}^{\infty} \rightarrow \mathfrak{M}_{1}^{\infty}\left(X_{0}\right)$ and $h_{1}: \mathbb{C P}^{\infty} \times \mathbb{C P}^{\infty} \rightarrow \mathfrak{M}_{1}^{\infty}\left(X_{1}\right)$ such that the following diagram

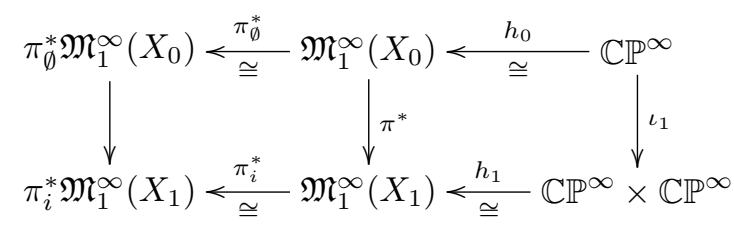

is homotopy commutative.

Proof. We will use the monad description of $\mathfrak{M}_{1}^{r}\left(X_{1}\right), \mathfrak{M}_{1}^{r}\left(X_{0}\right)$ (see appendix A). We define the following maps:

$$
\begin{array}{ll}
p_{0}: \mathfrak{M}_{1}^{r}\left(X_{0}\right) \rightarrow \mathbb{C P}^{r} & p_{0}:\left[a_{1}, a_{2}, b, c\right] \rightarrow[b] \\
p_{1}: \mathfrak{M}_{1}^{r}\left(X_{1}\right) \rightarrow \mathbb{C P}^{r} \times \mathbb{C P}^{r} & p_{1}:\left[a_{1}, a_{2}, d, b, c\right] \rightarrow\left([b],\left[\frac{\bar{c}^{t}}{\|c\|^{2}}\right]\right) \\
\Delta: \mathbb{C P}^{r} \rightarrow \mathbb{C P}^{r} \times \mathbb{C P}^{r} & \Delta:[u] \rightarrow([u],[u]) \\
f: \mathbb{C P}^{\infty} \times \mathbb{C P}^{\infty} \rightarrow \mathbb{C P}^{\infty} \times \mathbb{C P}^{\infty} & f:(x, y) \mapsto\left(x, x y^{-1}\right)
\end{array}
$$

where to define $f$ we observe that $\mathbb{C P}^{\infty}=B U(1)$ is homotopic to the free abelian group on $U(1)$. Now observe that the diagram

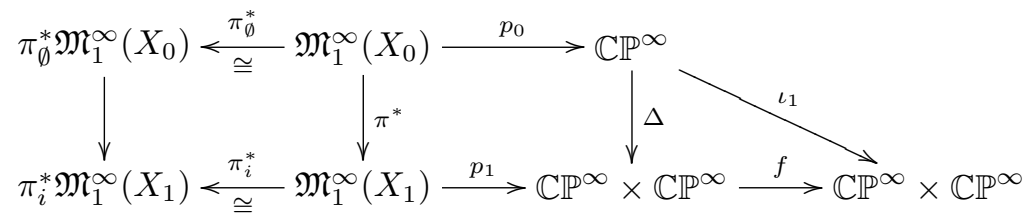

is homotopy commutative and the maps $p_{0}, p_{1}, f, \pi_{\emptyset}^{*}, \pi_{i}^{*}$ are homotopy equivalences. The statement of the lemma then follows by writing $h_{0}=p_{0}^{-1}$ and $h_{1}=p_{1}^{-1} f^{-1}$, where $p_{0}^{-1}, p_{1}^{-1}, f^{-1}$ are the homotopy inverses.

We are ready to prove theorem 3.1 . 
Proof of theorem 3.1. Let $C$ be the cone on $q$ points $v_{1}, \ldots, v_{q}$. Let

$$
M=\frac{\left(\coprod_{i=1}^{q} B U(1) \times B U(1) \times\left\{v_{i}\right\}\right) \amalg(B U(1) \times C)}{\left([u], v_{i}\right) \sim\left(\iota_{1}([u]), v_{i}\right)}
$$

We first show that $M$ is homotopically equivalent to $\mathfrak{M}_{1}^{\infty}\left(X_{q}\right)$.

Denote the points in $C$ by

$$
\left[t, v_{i}\right] \in C=\frac{[0,1] \times \coprod_{i}\left\{v_{i}\right\}}{\left(0, v_{i}\right) \sim\left(0, v_{j}\right) \sim *}
$$

Define a map

$$
\zeta:\left(\coprod_{i=1}^{q} B U(1) \times B U(1) \times\left\{v_{i}\right\}\right) \coprod(B U(1) \times C) \rightarrow \mathfrak{M}_{1}^{\infty}\left(X_{q}\right)
$$

as follows: denote the points in $C$ by $\left[t, v_{i}\right] \in C=\frac{[0,1] \times \coprod_{i}\left\{v_{i}\right\}}{\left(0, v_{i}\right) \sim\left(0, v_{j}\right) \sim *}$. Then define $\zeta$ by

$$
\begin{gathered}
B U(1) \times B U(1) \times\left\{v_{i}\right\} \ni\left(\left[u_{1}\right],\left[u_{2}\right], v_{i}\right) \mapsto \pi_{i}^{*} h_{1}\left(\left[u_{1}\right],\left[u_{2}\right]\right) \\
B U(1) \times C \ni\left([u],\left[t, v_{i}\right]\right) \mapsto \pi_{\emptyset}^{*} h_{0}([u]) \text { for } t<\frac{1}{3} \\
B U(1) \times C \ni\left([u],\left[t, v_{i}\right]\right) \mapsto \pi_{i}^{*} h_{1} \iota_{1}([u]) \text { for } t>\frac{2}{3}
\end{gathered}
$$

For $\frac{1}{3} \leq t \leq \frac{2}{3}$ use the homotopy between $\pi_{\emptyset}^{*} h_{0}$ and $p_{i}^{*} h_{1} \iota_{1}$ from lemma 3.2. $\zeta$ descends to the quotient to give a map $\zeta: M \rightarrow \mathfrak{M}_{1}^{\infty}\left(X_{q}\right)$. We want to apply Whitehead theorem to show $\zeta$ is a homotopy equivalence. The van Kampen theorem implies both $M$ and $\mathfrak{M}_{1}^{\infty}\left(X_{q}\right)$ are simply connected hence we only have to show $\zeta$ is an isomorphism in homology groups. We prove it by induction in $q^{\prime}=1, \ldots, q$. We apply the five lemma to the Meyer-Vietoris long exact sequence corresponding to open neighborhoods of the sets

$$
\begin{aligned}
& \pi_{q^{\prime}+1}^{*} \mathfrak{M}_{1}^{\infty}\left(X_{1}\right), \pi_{\left(1, \ldots, q^{\prime}\right)}^{*} \mathfrak{M}_{1}^{\infty}\left(X_{q^{\prime}}\right) \subset \mathfrak{M}_{1}^{\infty}\left(X_{q}\right) \\
& B U(1) \times B U(1) \times\left\{v_{i}\right\}, \bigcup_{l=1}^{q^{\prime}} B U(1) \times B U(1) \times\left\{v_{l}\right\} \subset M
\end{aligned}
$$

using the fact that the restrictions

$$
\begin{aligned}
& \zeta: B U(1) \times B U(1) \times\left\{v_{i}\right\} \rightarrow \pi_{i}^{*} \mathfrak{M}_{1}^{\infty}\left(X_{1}\right) \\
& \zeta: B U(1) \times C \rightarrow \pi_{\emptyset}^{*} \mathfrak{M}_{1}^{\infty}\left(X_{0}\right)
\end{aligned}
$$

are homotopy equivalences. It follows that $\zeta$ induces isomorphisms in all homology groups.

To conclude the proof we only have to show that $M$ is homotopically equivalent to

$$
B U(1) \times\left(\bigvee_{i=1}^{q} B U(1)\right)=\frac{\coprod_{l=1}^{q} B U(1) \times B U(1) \times\left\{v_{l}\right\}}{\left(x, *, v_{i}\right) \sim\left(x, *, v_{j}\right)}
$$

where $* \in B U(1)$ is the base point. Define an open cover of $B U(1) \times\left(\bigvee_{i} B U(1)\right)$ by $U_{i}=B U(1) \times B U(1) \times\left\{v_{i}\right\}$. Then the claim is a special case of proposition 4.1 in $[21]$. 


\section{AN OPEN COVER OF $\mathfrak{M}_{2}^{r}\left(X_{2}\right)$}

The objective of this section is to prove theorem 1.2. We begin by studying the case $q=2$. We will adopt, in this section and the next, the following notation: Denote the blow up points by $x_{L}, x_{R} \in X_{0}$. Let $\pi: X_{2} \rightarrow X_{0}$ be the blow up map at $x_{L}, x_{R}$. By abuse of notation we will denote by $\pi_{L}$ the maps $X_{2} \rightarrow X_{1}$ and $X_{1} \rightarrow X_{0}$ corresponding to the blow up at $x_{L}$ and in the same way $\pi_{R}$ will denote the blow up at $x_{R}$. We have the diagram

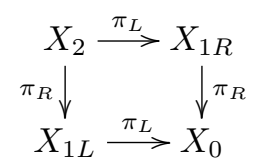

of blow up maps where $X_{1 L} \cong X_{1 R} \cong X_{1}$. Denote by $L_{L}$ and $L_{R}$ the exceptional divisors above $x_{L}$ and $x_{R}$ respectively. Again, by abuse of notation we identify $L_{L} \subset X_{2}$ with $L_{L} \subset X_{1 L}$ and the same for $L_{R}$. Write $x_{L}=\left[x_{1 L}, x_{2 L}, 1\right], x_{R}=$ $\left[x_{1 R}, x_{2 R}, 1\right], x_{L}, x_{R} \in X_{0}=\mathbb{C P}^{2}$. Since $x_{L} \neq x_{R}$ we may assume without loss of generality that $x_{1 L} \neq x_{1 R}$. Let $z_{i}=x_{i R}-x_{i L} . z_{1}, z_{2}$ determine a point $\left(\left[z_{1}, z_{2}, 1\right],\left[z_{1}, z_{2}\right]\right) \in X_{1} \backslash L_{\infty}=\widetilde{\mathbb{C P}^{2}} \backslash L_{\infty} \subset \mathbb{C P}^{2} \times \mathbb{C P}^{1}$. We are ready to state the first theorem of this section:

Theorem 4.1. Let

$$
\begin{aligned}
& A_{L}=\pi_{R}^{*} \mathfrak{M}_{2}^{r}\left(X_{1 L}\right)=\left\{\mathcal{E} \in \mathfrak{M}_{2}^{r}\left(X_{2}\right):\left.\mathcal{E}\right|_{L_{R}} \text { is trivial }\right\} \\
& A_{R}=\pi_{L}^{*} \mathfrak{M}_{2}^{r}\left(X_{1 R}\right)=\left\{\mathcal{E} \in \mathfrak{M}_{2}^{r}\left(X_{2}\right):\left.\mathcal{E}\right|_{L_{L}} \text { is trivial }\right\}
\end{aligned}
$$

and let $C=\mathfrak{M}_{2}^{r}\left(X_{2}\right) \backslash\left(A_{L} \cup A_{R}\right)$. Let $N_{L} \subset \mathfrak{M}_{2}^{r}\left(X_{1 L}\right)$ be the set of non-degenerate configurations $m=\left(a_{1}, a_{2}, d, b, c\right)$ such that the eigenvalues of $d a_{1}$ (equal to the eigenvalues of $a_{1} d$ ) are in a $\delta$ neighborhood of $0, z_{1}$. In a similar way define $N_{R} \subset$ $\mathfrak{M}_{2}^{r}\left(X_{1 R}\right)$. Let $N_{2}=\pi_{R}^{*} N_{L} \cup \pi_{L}^{*} N_{R} \cup C$. Then $\left\{A_{L}, A_{R}, N_{2}\right\}$ is an open cover of $\mathfrak{M}_{2}^{r}\left(X_{2}\right)$. There are homotopy equivalences
(1) $A_{L} \simeq A_{R} \simeq \mathfrak{M}_{2}^{r}\left(X_{1}\right)$
(2) $C \simeq \mathfrak{M}_{1}^{r}\left(X_{1}\right) \times \mathfrak{M}_{1}^{r}\left(X_{1}\right)$
(3) $A_{L} \cap A_{R} \simeq \mathfrak{M}_{2}^{r}\left(X_{0}\right)$
(4) $A_{L} \cap N_{2} \simeq N_{L} \simeq A_{R} \cap N_{2} \simeq N_{R} \simeq \mathfrak{M}_{1}^{r}\left(X_{1}\right) \times \mathfrak{M}_{1}^{r}\left(X_{0}\right)$
(5) $A_{L} \cap A_{R} \cap N_{2} \simeq \mathfrak{M}_{1}^{r}\left(X_{0}\right) \times \mathfrak{M}_{1}^{r}\left(X_{0}\right)$
(6) $N_{2} \simeq C$

From this open cover we get, in a standard way (see [21]), a spectral sequence:

Corollary 4.2. There is a spectral sequence converging to the cohomology of $\mathfrak{M}_{2}^{r}\left(X_{2}\right)$ with $E_{1}$ term

$$
\begin{aligned}
& E_{1}^{0, n}=H^{n}\left(A_{L}\right) \oplus H^{n}\left(A_{R}\right) \oplus H^{n}\left(N_{2}\right) \\
& E_{1}^{1, n}=H^{n}\left(A_{L} \cap A_{R}\right) \oplus H^{n}\left(A_{L} \cap N_{2}\right) \oplus H^{n}\left(A_{R} \cap N_{2}\right) \\
& E_{1}^{2, n}=H^{n}\left(A_{L} \cap A_{R} \cap N_{2}\right)
\end{aligned}
$$

In the next section we will study the $d_{1}$ differential of this spectral sequence.

We turn now to the proof of theorem 4.1. We will delay the proof that $N_{2}$ is open and begin by proving the homotopy equivalences (1), (2) and (3): 
Proposition 4.3. $A_{L}, A_{R}$ are open sets,

$$
C=\left\{[\mathcal{E}, \phi] \in \mathfrak{M}_{2}^{r}\left(X_{2}\right): c_{2}\left(\left(\pi_{i *} \mathcal{E}\right)^{\vee \vee}\right)=1, i=L, R\right\}
$$

and the following maps are isomorphisms $\left(\right.$ where $\left.\pi_{i *}^{\vee \vee}(\mathcal{E}) \stackrel{\text { def }}{=}\left(\pi_{i *} \mathcal{E}\right)^{\vee \vee}\right)$ :

$$
\begin{aligned}
& \pi_{R}^{*}: \mathfrak{M}_{2}^{r}\left(X_{1 L}\right) \rightarrow A_{L} \subset \mathfrak{M}_{2}^{r}\left(X_{2}\right) \\
& \pi_{L}^{*}: \mathfrak{M}_{2}^{r}\left(X_{1 R}\right) \rightarrow A_{R} \subset \mathfrak{M}_{2}^{r}\left(X_{2}\right) \\
& \pi_{R *}^{\vee \vee} \times \pi_{L *}^{\vee \vee}: C \rightarrow S_{0} \mathfrak{M}_{1}^{r}\left(X_{1 L}\right) \times S_{0} \mathfrak{M}_{1}^{r}\left(X_{1 R}\right) \\
& \pi^{*}: \mathfrak{M}_{2}^{r}\left(X_{0}\right) \rightarrow A_{L} \cap A_{R} \subset \mathfrak{M}_{2}^{r}\left(X_{2}\right)
\end{aligned}
$$

where $S_{0} \mathfrak{M}_{1}^{r}\left(X_{1}\right) \subset \mathfrak{M}_{1}^{r}\left(X_{1}\right)$ is the subspace of bundles $\mathcal{E}$ verifying $\left(\pi_{*} \mathcal{E}\right)^{\vee \vee}=\mathcal{O}_{X_{0}}^{r}$.

Proof. The isomorphisms for $A_{L}, A_{R}, A_{L} \cap A_{R}$ follows from theorem 2.1. That theorem also implies $A_{L}, A_{R}$ are open. It remains to look at the map $\pi_{R *}^{\vee \vee} \times$ $\pi_{L *}^{\vee \vee}: C \rightarrow S_{0} \mathfrak{M}_{1}^{r}\left(X_{1 L}\right) \times S_{0} \mathfrak{M}_{1}^{r}\left(X_{1 R}\right)$. The continuity of this map was proved in proposition 3.1 in [19]. We will construct an inverse for $\pi_{R *}^{\vee \vee} \times \pi_{L *}^{\vee \vee}$. Let $\left(\mathcal{E}_{L}, \phi_{L}\right) \in$ $S_{0} \mathfrak{M}_{1}^{r}\left(X_{1 L}\right),\left(\mathcal{E}_{R}, \phi_{R}\right) \in S_{0} \mathfrak{M}_{1}^{r}\left(X_{1 R}\right)$. Hartogs' theorem implies there are unique extensions of $\phi_{L}, \phi_{R}$ to maps

$$
\phi_{L}:\left.\mathcal{E}_{L}\right|_{X_{0} \backslash\left\{x_{L}\right\}} \rightarrow \mathcal{O}_{X_{0} \backslash\left\{x_{L}\right\}}^{r}, \phi_{R}:\left.\mathcal{E}_{R}\right|_{X_{0} \backslash\left\{x_{R}\right\}} \rightarrow \mathcal{O}_{X_{0} \backslash\left\{x_{R}\right\}}^{r}
$$

These maps induce an isomorphism $\mathcal{E}_{L} \cong \mathcal{E}_{R}$ over $X_{0} \backslash\left\{x_{L}, x_{R}\right\}$ which we use to glue $\mathcal{E}_{L}, \mathcal{E}_{R}$ and obtain a bundle $\mathcal{E} \rightarrow X_{2}$. The continuity of this map was proved in proposition 3.3 in [19]. This concludes the proof.

Before we continue we need a lemma. Let $\overline{\mathfrak{M}_{2}^{r}\left(X_{1}\right)}$ be the Donaldson-Uhlenbeck completion of the moduli space $\mathfrak{M}_{2}^{r}\left(X_{1}\right)$ (see the appendix). A blow-up $\pi: X_{2} \rightarrow$ $X_{1}$ induces a map $\pi_{*}: \mathfrak{M}_{2}^{r}\left(X_{2}\right) \rightarrow \mathfrak{M}_{2}^{r}\left(X_{1}\right)$ given by $\mathcal{E} \mapsto\left(\left(\pi_{*} \mathcal{E}\right)^{\vee \vee}, \ell\left(\left(\pi_{*} \mathcal{E}\right)^{\vee \vee} / \pi_{*} \mathcal{E}\right)\right)$

Lemma 4.4. Let $\mathcal{E}_{m} \in \overline{\mathfrak{M}_{2}^{r}\left(X_{1}\right)}$ and let $m=\left(a_{1}, a_{2}, d, b, c\right)$ be the configuration associated to $\mathcal{E}_{m}$. The following are equivalent:

(1) $\mathcal{E}_{m}$ is in the image of $\pi_{R *}: C \rightarrow \overline{\mathfrak{M}_{2}^{r}\left(X_{1}\right)}$;

(2) $c d b=0$ and the eigenvalues of $d a_{i}$ (equal to the ones of $a_{i} d$ ) are 0 and $z_{i}$;

(3) After a change of basis we can write

$$
a_{1}=\left[\begin{array}{cc}
a_{1}^{\prime} & 0 \\
0 & z_{1}
\end{array}\right], a_{2}=\left[\begin{array}{cc}
a_{2}^{\prime} & \frac{b^{\prime} c^{\prime \prime}}{z_{1}} \\
-\frac{b^{\prime \prime} c^{\prime}}{z_{1}} & z_{2}
\end{array}\right], d=\left[\begin{array}{ll}
0 & 0 \\
0 & 1
\end{array}\right], b=\left[\begin{array}{c}
b^{\prime} \\
b^{\prime \prime}
\end{array}\right], c=\left[\begin{array}{ll}
c^{\prime} & c^{\prime \prime}
\end{array}\right]
$$

with $c^{\prime \prime} b^{\prime \prime}=0$.

Proof. We will show that $1 \Rightarrow 2,2 \Rightarrow 3$ and $3 \Rightarrow 1$.

- $1 \Rightarrow 2$ : Suppose $\mathcal{E}_{m}=\pi_{R *} \tilde{\mathcal{E}}$ for some $\tilde{\mathcal{E}} \in C$. Then, by proposition 4.3, $\mathcal{E}_{m}^{\vee \vee} \in S_{0} \mathfrak{M}_{1}^{r}\left(X_{1 L}\right)$ and $\mathcal{E}_{m}$ is not locally free at the blow up point $x_{R}$. So, from proposition A.7, $\mathcal{E}_{m}^{\vee \vee}$ corresponds to a configuration of the form $\left[a_{1}^{\prime}, a_{2}^{\prime}, 0, b^{\prime}, c^{\prime}\right]$. Since $m$ is degenerate, by proposition A.5 after a change of basis it can be written in one of two forms, corresponding to the two types of special pairs. If $m$ is $b$-special then

$$
a_{i}=\left[\begin{array}{cc}
a_{i}^{\prime} & * \\
0 & a_{i}^{\prime \prime}
\end{array}\right], d=\left[\begin{array}{cc}
d^{\prime} & * \\
0 & d^{\prime \prime}
\end{array}\right], b=\left[\begin{array}{l}
b^{\prime} \\
0
\end{array}\right], c=\left[\begin{array}{ll}
c^{\prime} & c^{\prime \prime}
\end{array}\right]
$$


in which case the configuration is equivalent to the completely reducible configuration (see proposition A.5)

$$
\left(a_{1}^{\prime}, a_{2}^{\prime}, d^{\prime}, b^{\prime}, c^{\prime}\right) \oplus\left(a_{1}^{\prime \prime}, a_{2}^{\prime \prime}, d^{\prime \prime}, 0, c^{\prime \prime}\right)
$$

corresponding to an ideal bundle with singularity at $\left(a_{1}^{\prime \prime} d^{\prime \prime}, a_{2}^{\prime \prime} d^{\prime \prime}\right)$ and charge one bundle given by $\left(a_{1}^{\prime}, a_{2}^{\prime}, d^{\prime}, b^{\prime}, c^{\prime}\right)$. So we should have $d^{\prime}=0$ and $a_{i}^{\prime \prime} d^{\prime \prime}=$ $z_{i}$. Hence the eigenvalues of $d a_{i}$ are $0, z_{i}$ and $c d b=0$. A similar argument applies if $m$ is $c$-special.

- $2 \Rightarrow 3$ : Now assume the configuration $\left(a_{1}, a_{2}, d, b, c\right)$ satisfies 2. Fix a basis of eigenvectors $v_{0}, v_{1} \in V$ of $a_{1} d$ and $w_{0}, w_{1} \in W$ of $d a_{1}$ with $v_{0}, w_{0}$ corresponding to the eigenvalue 0 . Normalize $v_{1}, w_{1}$ so that $d v_{1}=w_{1}$. Then

$$
\begin{gathered}
a_{1}=\left[\begin{array}{cc}
a_{1}^{\prime} & 0 \\
0 & a_{1}^{\prime \prime}
\end{array}\right], a_{2}=\left[\begin{array}{cc}
a_{2}^{\prime} & \frac{b^{\prime} c^{\prime \prime}}{a_{1}^{\prime \prime}-d^{\prime} a_{1}^{\prime}} \\
\frac{b^{\prime \prime} c^{\prime}}{d^{\prime} a_{1}^{\prime}-a_{1}^{\prime \prime}} & a_{2}^{\prime \prime}
\end{array}\right] \\
d=\left[\begin{array}{ll}
d^{\prime} & 0 \\
0 & 1
\end{array}\right], b=\left[\begin{array}{l}
b^{\prime} \\
b^{\prime \prime}
\end{array}\right], c=\left[\begin{array}{ll}
c^{\prime} & c^{\prime \prime}
\end{array}\right]
\end{gathered}
$$

From $c d b=0$ we get $\left(b^{\prime} c^{\prime \prime}\right)\left(b^{\prime \prime} c^{\prime}\right)=0$. If $b^{\prime} c^{\prime \prime}=0$ then $a_{2}$ is lower triangular. If $b^{\prime \prime} c^{\prime}=0$ then $a_{2}$ is upper triangular. In both cases the diagonal entries of $a_{2} d$ are its eigenvalues. Hence, the condition about the eigenvalues of $a_{1} d$ and $a_{2} d$ yields the equations $a_{1}^{\prime} d^{\prime}=a_{2}^{\prime} d^{\prime}=0, a_{1}^{\prime \prime}=z_{1}$ and $a_{2}^{\prime \prime}=z_{2}$. Since $a_{1}(W)+a_{2}(W)+b\left(\mathbb{C}^{r}\right)=V$ we must have $d^{\prime}=0$.

- $3 \Rightarrow 1$ : Let $m=\left[a_{1}, a_{2}, d, b, c\right]$ be a configuration satisfying $3 . c^{\prime \prime} b^{\prime \prime}=0 \mathrm{im}$ plies either $c^{\prime \prime}=0$ or $b^{\prime \prime}=0$. It follows that the pair $(\operatorname{Span}\{(0,1)\}, \operatorname{Span}\{(0,1)\})$ is a special pair hence the configuration is degenerate. Now, from proposition A.5 it follows that $m$ is equivalent to the completely reducible configuration

$$
m^{\prime} \oplus m^{\prime \prime}=\left(a_{1}^{\prime}, a_{2}^{\prime}, 0, b^{\prime}, c^{\prime}\right) \oplus\left(z_{1}, z_{2}, 0,0\right)
$$

Notice that $\left(a_{1}^{\prime}, a_{2}^{\prime}, 0, b^{\prime}, c^{\prime}\right) \in S_{0} \mathfrak{M}_{1}^{r}\left(X_{1 L}\right)$. Then, from proposition 4.3, there is $\tilde{m} \in C$ such that $\pi_{R *} \tilde{m}^{\vee \vee}=m^{\prime}$. Then, from the characterization of points in the completion it follows that $\pi_{R *} \tilde{m}=m$.

Now we turn to the proof of homotopy equivalences (4) and (5) in theorem 4.1.

Definition 4.1. Let

$$
\begin{aligned}
& N_{z}=\left\{\left(a_{1 z}, a_{2 z}, b_{z}, c_{z}\right) \in \mathfrak{M}_{1}^{r}\left(X_{0}\right)|| a_{1 z}-z \mid<\delta\right\} \\
& N^{\prime}=\left\{\left(a_{1}^{\prime}, a_{2}^{\prime}, d^{\prime}, b^{\prime}, c^{\prime}\right) \in \mathfrak{M}_{1}^{r}\left(X_{1}\right)|| d^{\prime} a_{1}^{\prime} \mid<\delta\right\}
\end{aligned}
$$

Let $N_{0} \subset \mathfrak{M}_{2}^{r}\left(X_{0}\right)$ be the subset of points $\left(a_{1}, a_{2}, b, c\right)$ with the eigenvalues of $a_{1}$ lying in $\delta$ neighborhoods of $x_{L}$ and $x_{R}$. Then define the map $\boxplus_{0}: N_{x_{1 L}} \times N_{x_{1 R}} \rightarrow N_{0}$ by $\left[a_{1 L}, a_{2 L}, b_{L}, c_{L}\right] \boxplus_{0}\left[a_{1 R}, a_{2 R}, b_{R}, c_{R}\right]=\left[a_{1}, a_{2}, b, c\right]$ with

$$
a_{1}=\left[\begin{array}{cc}
a_{1 L} & 0 \\
0 & a_{1 R}
\end{array}\right], a_{2}=\left[\begin{array}{cc}
a_{2 L} & \frac{b_{L} c_{R}}{a_{1 R}-a_{1 L}} \\
\frac{b_{R} c_{L}}{a_{1 L}-a_{1 R}} & a_{2 R}
\end{array}\right], b=\left[\begin{array}{l}
b_{L} \\
b_{R}
\end{array}\right], c=\left[\begin{array}{ll}
c_{L} & c_{R}
\end{array}\right]
$$


and $\boxplus_{L}: N^{\prime} \times N_{z_{1}} \rightarrow N_{L}$ by $\left[a_{1}^{\prime}, a_{2}^{\prime}, d^{\prime}, b^{\prime}, c^{\prime}\right] \boxplus_{L}\left[a_{1}^{\prime \prime}, a_{2}^{\prime \prime}, b^{\prime \prime}, c^{\prime \prime}\right]=\left[a_{1}, a_{2}, d, b, c\right]$ with

$$
\begin{gathered}
a_{1}=\left[\begin{array}{cc}
a_{1}^{\prime} & 0 \\
0 & a_{1}^{\prime \prime}
\end{array}\right], a_{2}=\left[\begin{array}{cc}
a_{2}^{\prime} & \frac{b^{\prime} c^{\prime \prime}}{a_{1}^{\prime \prime}-d^{\prime} a_{1}^{\prime}} \\
\frac{b^{\prime \prime} c^{\prime}}{d^{\prime} a_{1}^{\prime}-a_{1}^{\prime \prime}} & a_{2}^{\prime \prime}
\end{array}\right] \\
d=\left[\begin{array}{cc}
d^{\prime} & 0 \\
0 & 1
\end{array}\right], b=\left[\begin{array}{c}
b^{\prime} \\
b^{\prime \prime}
\end{array}\right], c=\left[\begin{array}{ll}
c^{\prime} & c^{\prime \prime}
\end{array}\right]
\end{gathered}
$$

\section{Proposition 4.5.}

(1) The maps $\boxplus_{0}, \boxplus_{L}$ are homeomorphisms;

(2) The inclusions $N_{z} \rightarrow \mathfrak{M}_{1}^{r}\left(X_{0}\right), N^{\prime} \rightarrow \mathfrak{M}_{1}^{r}\left(X_{1}\right)$ are homotopy equivalences;

(3) $\pi_{R}^{*} N_{L} \cap \pi_{L}^{*} N_{R}=\pi_{\emptyset}^{*} N_{0}$.

Proof. Statement (2) is clear from the definition. To prove statement (3) we observe that

$$
\pi_{R}^{*} N_{L} \cap \pi_{L}^{*} N_{R}=\pi_{R}^{*} N_{L} \cap \pi_{\emptyset}^{*} \mathfrak{M}_{2}^{r}\left(X_{0}\right) \cong N_{L} \cap \pi_{L}^{*} \mathfrak{M}_{2}^{r}\left(X_{0}\right)
$$

The result now follows easily from proposition A.8. We turn to the proof of statement (1). It is an easy consequence of proposition A.5 that $\boxplus_{0}$ and $\boxplus_{L}$ preserve the nondegeneracy of the configurations so the maps are well defined.

Now we look at $\boxplus_{L}$. For $\delta$ small enough the eigenvalues of $a_{1} d$ are distinct. Hence we can choose, up to the action of $\left(\mathbb{C}^{*}\right)^{\times 4}$, eigenvector basis $\left\{v_{0}, v_{1}\right\} \subset V$ of $a_{1} d$ and $\left\{w_{0}, w_{1}\right\} \subset W$ of $d a_{1}$, where $v_{0}, w_{0}$ correspond to the eigenvalues near 0 . Normalize $v_{1}, w_{1}$ so that $d v_{1}=w_{1}$. Then the action of $\left(\mathbb{C}^{*}\right)^{\times 4}$ is reduced to an action of $\left(\mathbb{C}^{*}\right)^{\times 3}$. We can thus write (see also equation (3))

$$
\begin{gathered}
a_{1}=\left[\begin{array}{cc}
a_{1}^{\prime} & 0 \\
0 & a_{1}^{\prime \prime}
\end{array}\right], a_{2}=\left[\begin{array}{cc}
a_{2}^{\prime} & \frac{b^{\prime} c^{\prime \prime}}{a_{1}^{\prime \prime}-d^{\prime} a_{1}^{\prime}} \\
\frac{b^{\prime \prime} c^{\prime}}{d^{\prime} a_{1}^{\prime}-a_{1}^{\prime \prime}} & a_{2}^{\prime \prime}
\end{array}\right] \\
d=\left[\begin{array}{ll}
d^{\prime} & 0 \\
0 & 1
\end{array}\right], b=\left[\begin{array}{l}
b^{\prime} \\
b^{\prime \prime}
\end{array}\right], c=\left[\begin{array}{ll}
c^{\prime} & c^{\prime \prime}
\end{array}\right]
\end{gathered}
$$

The group $\left(\mathbb{C}^{*}\right)^{\times 3}$ acts transitively on equivalence classes of such configurations written in the above canonical form. This shows the existence of an inverse, hence $\boxplus_{L}$ is a homeomorphism. The proof for $\boxplus_{0}$ is similar.

We will need the following identity:

Proposition 4.6. Let $\tau: \mathfrak{M}_{k}^{r}\left(X_{0}\right) \rightarrow \mathfrak{M}_{k}^{r}\left(X_{0}\right)$ be defined by

$$
\tau\left(a_{1}, a_{2}, b, c\right)=\left(a_{1}-x_{1 L} \mathbb{1}, a_{2}-x_{2 L} \mathbb{1}, b, c\right)
$$

Let $m_{1}, m_{2} \in \mathfrak{M}_{1}^{r}\left(X_{0}\right)$. Then $\pi_{L}^{*}\left(m_{1} \boxplus_{0} m_{2}\right)=\pi_{L}^{*} m_{1} \boxplus_{L} \tau\left(m_{2}\right)$.

Proof. It follows easily from proposition A.8.

The maps $\boxplus_{0}, \boxplus_{L}$ extend to the closure $\bar{N}^{\prime}, \bar{N}_{z}$ of $N^{\prime}, N_{z}$. The following proposition is a direct consequence of proposition A.5:

\section{Proposition 4.7.}

- Let $m_{L}=\left[a_{1 L}, a_{2 L}, b_{L}, c_{L}\right] \in \bar{N}_{x_{1 L}}, m_{R}=\left[a_{1 R}, a_{2 R}, b_{R}, c_{R}\right] \in \bar{N}_{x_{1 R}}$. Then the following are equivalent:

(1) $m_{L} \boxplus_{0} m_{R}$ is degenerate;

(2) Either $m_{L}$ or $m_{R}$ is degenerate.

(3) At least one of the 4 vectors $b_{L}, b_{R}, c_{L}, c_{R}$ is zero. 
- Let $m^{\prime}=\left[a_{1}^{\prime}, a_{2}^{\prime}, d^{\prime}, b^{\prime}, c^{\prime}\right] \in \bar{N}^{\prime}, m^{\prime \prime}=\left[a_{1}^{\prime \prime}, a_{2}^{\prime \prime}, b^{\prime \prime}, c^{\prime \prime}\right] \in \bar{N}_{z_{1}}$. The following are equivalent:

(1) $m^{\prime} \boxplus_{L} m^{\prime \prime}$ is degenerate;

(2) Either $m^{\prime}$ or $m^{\prime \prime}$ is degenerate;

(3) One of the 4 vectors $b^{\prime}, b^{\prime \prime}, c^{\prime}, c^{\prime \prime}$ is zero.

We are ready to prove

Proposition 4.8. $N_{2}$ is an open neighborhood of $C$.

Proof. From lemma 4.4 it follows immediately that $\pi_{R *} C \subset \bar{N}_{L}$.

Suppose there is a sequence $y_{n} \in \mathfrak{M}_{2}^{r}\left(X_{1 L}\right)$ such that $y_{n} \rightarrow y \in \pi_{R *} C$. Write $y_{n}=\left[a_{1 n}, a_{2 n}, d_{n}, b_{n}, c_{n}\right]$. Then, by property 2 in lemma 4.4 the eigenvalues of $d_{n} a_{i n}$ converge to $0, z_{i}$. Hence, for $n$ large enough $y_{n} \in N_{L}$. Hence $N_{L} \cup \pi_{R *} C$ is an open neighborhood of $\pi_{R *} C$.

Suppose there is a sequence $x_{n} \rightarrow x \in C$ such that $x_{n} \notin N_{2}$. Hence $x_{n} \notin C$ so, by passing to a subsequence we may assume without loss of generality that $x_{n} \in \pi_{R}^{*} \mathfrak{M}_{2}^{r}\left(X_{1 L}\right)$. Let $y_{n}=\pi_{R *} x_{n} \in \mathfrak{M}_{2}^{r}\left(X_{1 L}\right)$ and write $y_{n}=\left[a_{1 n}, a_{2 n}, d_{n}, b_{n}, c_{n}\right]$. Then $y_{n} \rightarrow y=\pi_{R *} x$ by continuity of $\pi_{R *}$, and $y_{n} \notin N_{L}$. But by property 2 in lemma 4.4 the eigenvalues of $d_{n} a_{i n}$ converge to $0, z_{i}$ which implies, for $n$ large enough, that $y_{n} \in N_{L}$.

Finaly we prove the homotopy equivalence (6):

Proposition 4.9. The inclusion $C \rightarrow N_{2}$ is a strong deformation retract.

Proof. We will construct a homotopy $H_{2}: N_{2} \times[0,1] \rightarrow N_{2}$ betwen the identity and a retraction $N_{2} \rightarrow C$. Let $H_{x_{1}, x_{2}}: \bar{N}_{z} \times[0,1] \rightarrow \bar{N}_{z}$ be defined by

$$
H_{x_{1}, x_{2}}\left(a_{1}, a_{2}, b, c, t\right)=\left(t^{2} a_{1}+\left(1-t^{2}\right) x_{1}, t^{2} a_{2}+\left(1-t^{2}\right) x_{2}, t b, t c\right)
$$

and let $H_{1}: \bar{N}^{\prime} \times[0,1] \rightarrow \bar{N}^{\prime}$ be defined by

$$
H_{1}\left(a_{1}^{\prime}, a_{2}^{\prime}, d^{\prime}, b^{\prime}, c^{\prime}, t\right)=\left(a_{1}^{\prime}, a_{2}^{\prime}, t^{2} d^{\prime}, b^{\prime}, c^{\prime}\right)
$$

Then we defined $H_{L}: \bar{N}_{L} \times[0,1] \rightarrow \bar{N}_{L}$ by

$$
H_{L}\left(m^{\prime} \boxplus_{L} m^{\prime \prime}, t\right) \stackrel{\text { def }}{=} H_{1}\left(m^{\prime}, t\right) \boxplus_{L} H_{z_{1}, z_{2}}\left(m^{\prime \prime}, t\right)
$$

We define $H_{2}$ as the unique solution of the system of equations

$$
\begin{aligned}
& \pi_{R *} H_{2}(x, t)=H_{L}\left(\pi_{R *} x, t\right) \\
& \pi_{L *} H_{2}(x, t)=H_{R}\left(\pi_{L *} x, t\right)
\end{aligned}
$$

We have to show existence and uniqueness of solution. Then we will show that $H_{2}$ defines a homotopy between the identity on $N_{2}$ and a retraction $N_{2} \rightarrow C$. We define the auxiliary map $H_{0}: \bar{N}_{0} \times[0,1] \rightarrow \bar{N}_{0}$ by

$$
H_{0}\left(m_{L} \boxplus_{0} m_{R}, t\right) \stackrel{\text { def }}{=} H_{x_{1 L}, x_{2 L}}\left(m_{L}, t\right) \boxplus_{0} H_{x_{1 R}, x_{2 R}}\left(m_{R}, t\right)
$$

To prove existence and uniqueness of solution of the system (7) we consider two cases:

(1) Assume that either $t=0$ or $x \in C$. Then we claim that $H_{L}\left(\pi_{R *} x, t\right) \in$ $\pi_{R *} C, H_{R}\left(\pi_{L *} x, t\right) \in \pi_{L *} C$. If $t=0$ this follows directly from lemma 4.4. If $x \in C$ then, from lemma 4.4 we can write

$$
\pi_{R *} x=x^{\prime} \boxplus_{L} x^{\prime \prime}=\left(a_{1}^{\prime}, a_{2}^{\prime}, 0, b^{\prime}, c^{\prime}\right) \boxplus_{L}\left(a_{1}^{\prime \prime}, a_{2}^{\prime \prime}, b^{\prime \prime}, c^{\prime \prime}\right)
$$


with $c^{\prime \prime} b^{\prime \prime}=0$. It then follows from the definition of $H_{L}$ that $H_{L}\left(\pi_{R *} x, t\right)=$ $\pi_{R *} x$ for all $t$. In the same way we see that $H_{R}\left(\pi_{L *} x, t\right)=\pi_{L *} x$. This proves the claim. Then, existence and uniqueness follows from proposition 4.3.

(2) Assume $t \neq 1$ and $x \notin C$. Then we may assume $\pi_{R *} x \in N_{L}$. Then, since $H_{L}\left(\pi_{R *} x, t\right) \in N_{L}$, we get from $(7)$

$$
\pi_{R *} H_{2}(x, t)=H_{L}\left(\pi_{R *} x, t\right) \Rightarrow H_{2}(x, t)=\pi_{R}^{*} H_{L}\left(\pi_{R *} x, t\right)
$$

This proves uniqueness. To prove existence we need to show that

$$
\pi_{L *} H_{2}(x, t)=\pi_{L *} \pi_{R}^{*} H_{L}\left(\pi_{R *} x, t\right)=H_{R}\left(\pi_{L *} x, t\right)
$$

It is enough to show this for the case where $x=\pi_{L}^{*} \pi_{R}^{*} y$ for some $y \in$ $N_{0}$ since the set of points of this form is dense and $H_{L}, H_{R}, \pi_{L *}, \pi_{R}^{*}, \pi_{R *}$ are continuous. It is an easy computation to show that $H_{L}\left(\pi_{L}^{*} y, t\right)=$ $\pi_{L}^{*} H_{0}(y, t), H_{R}\left(\pi_{R}^{*} y, t\right)=\pi_{R}^{*} H_{0}(y, t)$. It follows that

$$
\pi_{L *} \pi_{R}^{*} H_{L}\left(\pi_{R *} x, t\right)=\pi_{L *} \pi_{R}^{*} \pi_{L}^{*} H_{0}(y, t)=\pi_{R}^{*} H_{0}(y, t)=H_{R}\left(\pi_{L *} x, t\right)
$$

Now we need to show that $H_{2}$ is the desired homotopy. Direct inspection shows $H_{2}(x, 1)=x$. We saw in (1) above that, for $x \in C, H_{2}(x, t)=x$ and $H_{2}(x, 0) \in C$. The continuity of $H_{2}$ follows from the continuity of $\pi_{L *}, \pi_{R *}, H_{L}, H_{R}$ : let $\left(x_{n}, t_{n}\right) \rightarrow$ $(x, t) \in N_{2}$. Then, after passing to a subsequence, we get $H_{2}\left(x_{n_{k}}, t_{n_{k}}\right) \rightarrow(\tilde{x}, \tilde{t}) \in$ $\bar{N}_{2}$. Then equations 7 imply that $(\tilde{x}, \tilde{t}) \in N_{2}$ and unicity of solution implies $(\tilde{x}, \tilde{t})=$ $H_{2}(x, t)$. Applying this reasoning to every sublimit of $H_{2}\left(x_{n}, t_{n}\right)$ we conclude that $H_{2}\left(x_{n}, t_{n}\right) \rightarrow H_{2}(x, t)$. Hence $H_{2}$ is continuous.

We now prove the general case (theorem 1.2). What remains to be proven is:

Theorem 4.10. Let $A_{i}, A_{\emptyset}$ be as in theorem 2.1 and let $N_{i j}=\pi_{i j}^{*} N_{2}$. Then

(1) $\left\{A_{i}\right\} \cup\left\{N_{i j}\right\}$ is an open cover of $\mathfrak{M}_{2}^{r}\left(X_{q}\right)$;

(2) There are homotopy equivalences

$$
N_{i j} \cap A_{i} \simeq \mathfrak{M}_{1}^{r}\left(X_{1}\right) \times \mathfrak{M}_{1}^{r}\left(X_{0}\right) \quad N_{i j} \cap A_{\emptyset} \simeq \mathfrak{M}_{1}^{r}\left(X_{0}\right) \times \mathfrak{M}_{1}^{r}\left(X_{0}\right)
$$

(3) For $k \notin\{i, j\}, N_{i j} \cap A_{k}=N_{i j} \cap A_{\emptyset}$;

(4) For different sets $\{i, j\} \neq\{k, l\}, N_{i j} \cap N_{k l}=\emptyset$.

Proof. Statement (1) follows from theorems 2.1 and 4.1. Statement (2) follows from proposition 2.2. To prove statement (3) observe that $N_{i j} \cap A_{k}=N_{i j} \cap A_{i j} \cap A_{k}$. So we turn to statement (4). First we look at $N_{i j} \cap N_{j k}$. Let $x_{1}, \ldots, x_{q} \subset \mathbb{C P}^{2}$ be the blow-up points and write $x_{i}=\left[x_{1 i}, x_{2 i}, 1\right]$. We may assume without loss of generality that the balls $B_{\delta}\left(x_{1 i}\right)$ are disjoint. Now observe that

$$
N_{i j} \cap N_{j k}=N_{i j} \cap A_{i j} \cap A_{j k} \cap N_{j k}=N_{i j} \cap A_{j} \cap N_{j k}
$$

Let $z_{i}=x_{1 i}-x_{1 j}$ and let $z_{k}=x_{1 k}-x_{1 j}$. Then, theorem 4.1 states that $N_{i j} \cap A_{j}$ is the set of non-degenerate configurations $\left(a_{1}, a_{2}, d, b, c\right)$ such that the eigenvalues of $d a_{1}$ are in a $\delta$ neighborhood of 0 and $z_{i}$. Hence $N_{i j} \cap A_{j}$ and $N_{j k} \cap A_{j}$ are disjoint. This shows $N_{i j} \cap N_{j k}=\emptyset$. The case $N_{i j} \cap N_{k l}$ for $\{i, j\} \cap\{k, l\}=\emptyset$ is treated similarly. 


\section{The DIFFERENTIAL $d_{1}$}

The objective of this section is to obtain, in the limit when $r \rightarrow \infty$, the homotopy type of the inclusion maps (see theorem 4.1)

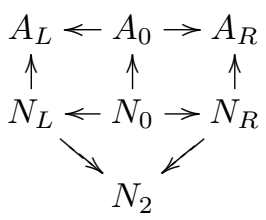

where $A_{0}=A_{L} \cap A_{R}=\pi_{\emptyset}^{*} \mathfrak{M}_{2}^{r}\left(X_{0}\right)$. Since these spaces are classifying spaces it is enough to study the pullback under these maps of the tautological bundles. This will allow us to compute the $d_{1}$ differential in the spectral sequence introduced in corolary 4.2, from where we will compute the homology of $\mathfrak{M}_{2}^{\infty}\left(X_{2}\right)$.

Definition 5.1. Let

$$
\begin{aligned}
& \tilde{F}_{0}(k, r)=\frac{\left\{(u, v) \in \operatorname{Hom}\left(\mathbb{C}^{k}, \mathbb{C}^{r}\right) \times \operatorname{Hom}\left(\mathbb{C}^{k}, \mathbb{C}^{r}\right) \mid u, v \text { are injective }\right\}}{(u, v) \sim\left(u\left(\bar{g}^{t}\right)^{-1}, v g\right), g \in G l(k, \mathbb{C})} \\
& \tilde{F}_{1}(k, r)=\frac{\left\{(u, v) \in \operatorname{Hom}\left(\mathbb{C}^{k}, \mathbb{C}^{r}\right) \times \operatorname{Hom}\left(\mathbb{C}^{k}, \mathbb{C}^{r}\right) \mid u, v \text { are injective }\right\}}{(u, v) \sim\left(u\left(\bar{g}_{u}^{t}\right)^{-1}, v g_{v}\right), g_{u}, g_{v} \in G l(k, \mathbb{C})}
\end{aligned}
$$

and define $F_{0}(k, r) \subset \tilde{F}_{0}(k, r)$ and $F_{1}(k, r) \subset \tilde{F}_{1}(k, r)$ by

$$
\begin{aligned}
& F_{0}(k, r)=\left\{\left[\bar{b}^{t}, c\right] \in \tilde{F}_{0}(k, r), b c=0\right\} \\
& F_{1}(k, r)=\left\{\left[\bar{b}^{t}, c\right] \in \tilde{F}_{1}(k, r), b c=0\right\}
\end{aligned}
$$

Lemma 5.1. Let $j_{0}: F_{0}(k, r) \rightarrow \mathfrak{M}_{k}^{r}\left(X_{0}\right), j_{1}: F_{1}(k, r) \rightarrow \mathfrak{M}_{k}^{r}\left(X_{1}\right)$ be the inclusion maps given by $\left[\bar{b}^{t}, c\right] \mapsto[b, c]$. Then we have the homotopy commutative diagram

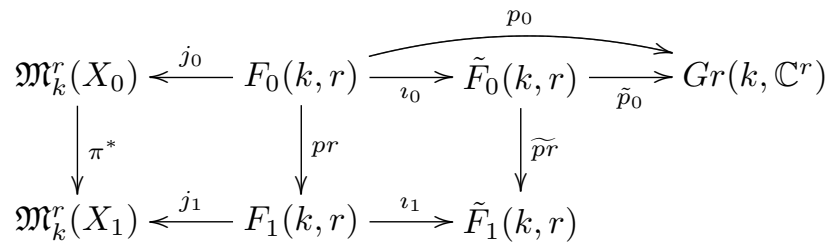

where $p_{0}$ is the projection $[b, c] \mapsto[c]$. Moreover, in the rank stable limit, the maps $\imath_{0}, \imath_{1}, p_{0}, \tilde{p}_{0}, j_{0}, j_{1}$ are homotopy equivalences.

Proof. We divide the proof into three steps:

(1) $p_{0}, \tilde{p}_{0}$ are fibrations with fibers $M(k, r-k)$ and $M(k, r)$ respectively where $M(k, r)=\frac{U(r)}{U(k)}$ is the space of injective maps from $\mathbb{C}^{k}$ to $\mathbb{C}^{r}$ which is contractible in the stable range. That proves $p_{0}, \tilde{p}_{0}$ are homotopy equivalences. It imediatelly follows that $\imath_{0}$ is a homotopy equivalence.

(2) Now we look at $\imath_{1}$. Consider the projection $p_{1}: F_{1}(k, r) \rightarrow G r\left(k, \mathbb{C}^{r}\right)$ given by $[b, c] \mapsto[c]$. When $r \rightarrow \infty$, the spectral sequence associated with the fibration $p_{1}: F_{1}(k, r) \rightarrow G r\left(k, \mathbb{C}^{r}\right)$ (whose fiber is $\operatorname{Gr}\left(k, \mathbb{C}^{r-k}\right)$ ) collapses since all homology is in even dimensions. It easilly follows that $\imath_{1}$ is an isomorphism in all homology groups, hence an homotopy equivalence. 
(3) Finally we need to prove the statements about $j_{0}, j_{1}$. First we look at $j_{0}$. Let $\mathcal{R}$ be the space of configurations $\left(a_{1}, a_{2}, b, c\right)$ and let $\mathcal{R}^{F} \subset \mathcal{R}$ be the subspace configurations of the form $(0,0, b, c)$. Then we have the fibration map

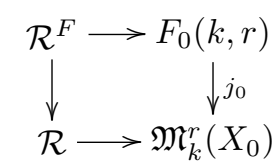

In the rank stable limit the spaces $\mathcal{R}^{F}$ and $\mathcal{R}$ are contractible (see [18], [2]) so, by the five lemma $j_{0}$ is an isomorphism in homotopy groups hence an homotopy equivalence. A similar proof works for $j_{1}$.

Now we turn to the main theorem of this section.

Definition 5.2. Let $E, L$ be the tautological bundles over $\operatorname{Gr}(2, \infty)$ and $\operatorname{Gr}(1, \infty)$ respectivelly. Consider the compositions

$$
\begin{aligned}
& A_{0} \stackrel{\pi_{\emptyset *}}{\longrightarrow} \mathfrak{M}_{2}^{\infty}\left(X_{0}\right) \stackrel{j_{0}^{-1}}{\longrightarrow} F_{0}(2, \infty) \stackrel{p_{0}}{\longrightarrow} \operatorname{Gr}\left(2, \mathbb{C}^{\infty}\right) \\
& N_{0} \stackrel{\boxplus_{0}^{-1}}{\longrightarrow} N_{x_{1 L}} \times N_{x_{1 R}} \stackrel{p_{L}}{\longrightarrow} N_{x_{1 L}} \longrightarrow \mathfrak{M}_{1}^{\infty}\left(X_{0}\right) \stackrel{p_{0} j_{0}^{-1}}{\longrightarrow} \operatorname{Gr}\left(1, \mathbb{C}^{\infty}\right) \\
& N_{0} \stackrel{\boxplus_{0}^{-1}}{\longrightarrow} N_{x_{1 L}} \times N_{x_{1 R}} \stackrel{p_{R}}{\longrightarrow} N_{x_{1 R}} \longrightarrow \mathfrak{M}_{1}^{\infty}\left(X_{0}\right) \stackrel{p_{0} j_{0}^{-1}}{\longrightarrow} \operatorname{Gr}\left(1, \mathbb{C}^{\infty}\right) \\
& N_{L} \stackrel{\boxplus_{L}^{-1}}{\longrightarrow} N^{\prime} \times N_{z_{1}} \stackrel{p^{\prime \prime}}{\longrightarrow} N_{z_{1}} \longrightarrow \mathfrak{M}_{1}^{\infty}\left(X_{0}\right) \stackrel{p_{0} j_{0}^{-1}}{\longrightarrow} \operatorname{Gr}\left(1, \mathbb{C}^{\infty}\right)
\end{aligned}
$$

Then we define the following bundles:

- $E_{0} \rightarrow A_{0}$ is the pullback of $E$ under the composition 9 .

- $L_{0 L, 0} \rightarrow N_{0}$ is the pullback of $L$ under 10

- $L_{0 R, 0} \rightarrow N_{0}$ is the pullback of $L$ under 11

- $L_{0 R, L} \rightarrow N_{L}$ is the pullback of $L$ under 12

Now let $\tilde{E}_{u}, \tilde{E}_{v} \rightarrow \tilde{F}_{1}(2, r)$ be the tautological bundles corresponding to $u, v$ and let $\tilde{L}_{u}, \tilde{L}_{v}$ be the tautological line bundles over $\tilde{F}_{1}(1, \infty)$. Consider the compositions

$$
\begin{aligned}
& A_{L} \stackrel{\pi_{R *}}{\longrightarrow} \mathfrak{M}_{2}^{\infty}\left(X_{1 L}\right) \stackrel{j_{1}^{-1}}{\longrightarrow} F_{1}(2, \infty) \stackrel{\imath_{1}}{\longrightarrow} \tilde{F}_{1}(2, \infty) \\
& N_{L} \stackrel{\boxplus_{L}^{-1}}{\longrightarrow} N^{\prime} \times N_{z_{1}} \stackrel{p^{\prime}}{\longrightarrow} N^{\prime} \longrightarrow \mathfrak{M}_{1}^{\infty}\left(X_{1}\right) \stackrel{\imath_{1} j_{1}^{-1}}{\longrightarrow} \tilde{F}_{1}(1, \infty) \\
& N_{2} \stackrel{\simeq}{\longrightarrow} C \stackrel{\pi_{L *}^{\vee \vee}}{\longrightarrow} S_{0} \mathfrak{M}_{1}^{\infty}\left(X_{1 L}\right)>\mathfrak{M}_{1}^{\infty}\left(X_{1 L}\right) \stackrel{\imath_{1} j_{1}^{-1}}{\longrightarrow} \tilde{F}_{1}(1, \infty) \\
& N_{2} \stackrel{\simeq}{\longrightarrow} C \stackrel{\pi_{R *}^{\vee \vee}}{\longrightarrow} S_{0} \mathfrak{M}_{1}^{\infty}\left(X_{1 R}\right) \longrightarrow \mathfrak{M}_{1}^{\infty}\left(X_{1 R}\right) \stackrel{{ }_{1} j_{1}^{-1}}{\longrightarrow} \tilde{F}_{1}(1, \infty)
\end{aligned}
$$

We define the bundles

- $E_{b L}, E_{c L} \rightarrow A_{L}$ are the pullback of $\tilde{E}_{u}, \tilde{E}_{v}$ under 13 .

- $L_{b L, L}, L_{c L, L} \rightarrow N_{L}$ are the pullback of $\tilde{L}_{u}, \tilde{L}_{v}$ under 14

- $L_{b L, 2}, L_{c L, 2} \rightarrow N_{2}$ are the pullback of $\tilde{L}_{u}, \tilde{L}_{v}$ under 15

- $L_{b R, 2}, L_{c R, 2} \rightarrow N_{2}$ are the pullback of $\tilde{L}_{u}, \tilde{L}_{v}$ under 16

Theorem 5.2. We have the following bundle isomorphisms: 
(1) $\left.E_{b L}\right|_{A_{0}}=E_{0},\left.E_{c L}\right|_{A_{0}}=E_{0}$

(2) $\left.L_{b L, L}\right|_{N_{0}} \cong L_{0 L, 0},\left.L_{c L, L}\right|_{N_{0}} \cong L_{0 L, 0},\left.L_{0 R, L}\right|_{N_{0}} \cong L_{0 R, 0}$

(3) $\left.E_{b L}\right|_{N_{L}} \cong L_{b L} \oplus L_{0 R},\left.E_{c L}\right|_{N_{L}} \cong L_{c L} \oplus L_{0 R}$

(4) $\left.E_{0}\right|_{N_{0}} \cong L_{0 L, 0} \oplus L_{0 R, 0}$.

(5) $\left.\left.L_{b R, 2}\right|_{N_{L}} \cong L_{c R, 2}\right|_{N_{L}} \cong L_{0 R, L}$

(6) $\left.L_{b L, 2}\right|_{N_{L}} \cong L_{b L, L},\left.L_{c L, 2}\right|_{N_{L}} \cong L_{c L, L}$

Similar statements hold for the spaces $A_{R}, N_{R}$ and the maps $N_{R} \rightarrow A_{R}, N_{R} \rightarrow N_{2}$ and $N_{0} \rightarrow N_{R}$.

Proof.

(1) First we show that $\left.\left.E_{b L}\right|_{A_{0}} \cong E_{c L}\right|_{A_{0}} \cong E_{0}$. Consider diagram (8). We will start by defining a homotopy inverse $q: G r\left(k, \mathbb{C}^{r}\right) \rightarrow \tilde{F}_{0}(k, r)$ to the map $\tilde{p}_{0}: \tilde{F}_{0} \rightarrow G r\left(k, \mathbb{C}^{\infty}\right)$ as follows: choose a map $c: \mathbb{C}^{k} \rightarrow \mathbb{C}^{r}$ representing an element $[c] \in G r\left(k, \mathbb{C}^{r}\right)$. Choose $h \in G l(k, \mathbb{C})$ such that $c h$ is orthogonal. Then define $q([c])=[c h, c h]$. This map is well defined and independent of the choice of $h$. Also $p_{0} q=\mathbb{1}$ hence $p_{0}=q^{-1}$. Now observe that the composition

$$
\tilde{p r} \circ q: G r\left(k, \mathbb{C}^{r}\right) \rightarrow \tilde{F}_{1}(k, r)=\operatorname{Gr}\left(k, \mathbb{C}^{r}\right) \times G r\left(k, \mathbb{C}^{r}\right)
$$

is the diagonal map. It follows that, if $E$ is the tautological bundle over $\operatorname{Gr}\left(k, \mathbb{C}^{\infty}\right)$, then

$$
q^{*} \tilde{p r} \tilde{E}_{u} \cong q^{*} \tilde{p r}^{*} \tilde{E}_{v} \cong E
$$

To show that $\left.\left.E_{b L}\right|_{A_{0}} \cong E_{c L}\right|_{A_{0}} \cong E_{0}$ it suffices to show that $p r^{*} \imath_{1}^{*} \tilde{E}_{u} \cong$ $p r^{*} \imath_{1}^{*} \tilde{E}_{v} \cong p_{0}^{*} E$. We have

$$
p r^{*} \imath_{1}^{*} \tilde{E}_{u}=\imath_{0}^{*} \widetilde{p r}^{*} \tilde{E}_{u} \cong p_{0}^{*} q^{*} \widetilde{p r}^{*} \tilde{E}_{u}=p_{0}^{*} E
$$

and a similar statement is true for $\tilde{E}_{v}$. This concludes the proof.

(2) We want to show that $\left.L_{b L, L}\right|_{N_{0}} \cong L_{0 L, 0},\left.L_{c L, L}\right|_{N_{0}} \cong L_{0 L, 0}$ and $\left.L_{0 R, L}\right|_{N_{0}} \cong$ $L_{0 R, 0}$. We have the commutative diagram (see proposition 4.6)

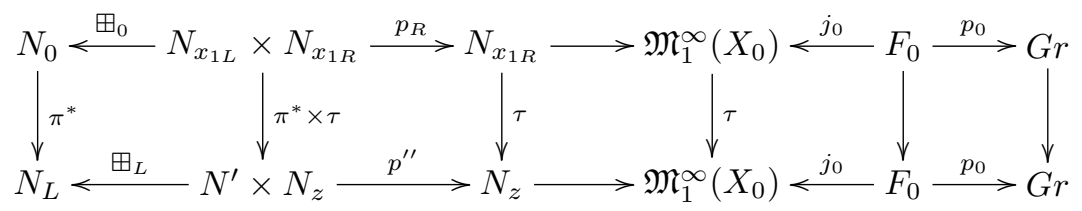

from which it follows that $\left.L_{0 R, L}\right|_{N_{0}} \cong L_{0 R, 0}$. We also have the commutative diagram

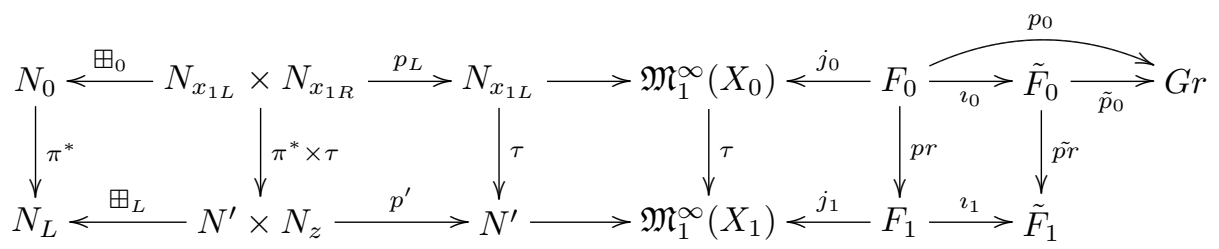

from which it follows, as in step (1), that $\left.\left.L_{b L, L}\right|_{N_{0}} \cong L_{c L, L}\right|_{N_{0}} \cong L_{0 L, 0}$. 
(3) We want to show that $\left.E_{b L}\right|_{N_{L}} \cong L_{b L, L} \oplus L_{0 R, L}$ and $\left.E_{c L}\right|_{N_{L}} \cong L_{c L, L} \oplus L_{0 R, L}$. Consider the following diagram:

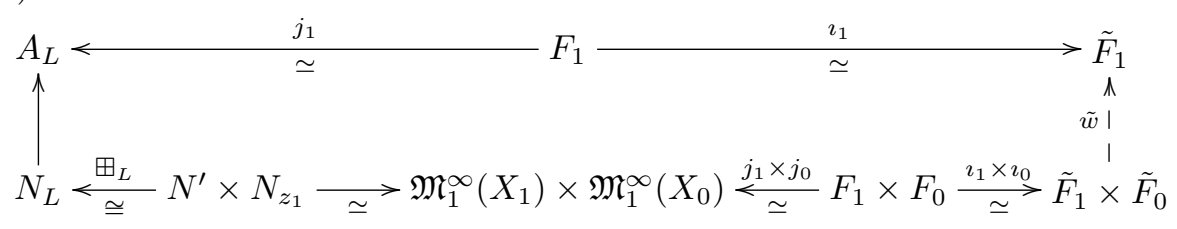

Since $\tilde{p r}^{*} \tilde{L}_{u} \cong \tilde{p} r^{*} \tilde{L}_{v} \cong \tilde{p}_{0}^{*} L$, the proof will be complete if we show there is a map $\tilde{w}: \tilde{F}_{1}(1, \infty) \times \tilde{F}_{0}\left(1, \mathbb{C}^{\infty}\right) \rightarrow \tilde{F}_{1}(2, \infty)$ making the diagram homotopy commutative, such that

$$
\tilde{w}^{*} \tilde{E}_{u}=\tilde{L}_{u} \oplus \tilde{p r} \tilde{L}_{u}, \tilde{w}^{*} \tilde{E}_{v}=\tilde{L}_{v} \oplus \tilde{p r}^{*} \tilde{L}_{v}
$$

We begin by building $\tilde{w}$. Define maps $s_{L}, s_{R}: G r\left(1, \mathbb{C}^{\infty}\right) \rightarrow G r\left(1, \mathbb{C}^{\infty}\right)$ as follows: let $v: \mathbb{C} \rightarrow \mathbb{C}^{\infty}$ and write $v=\left(v^{1}, v^{2}, \ldots\right)$. Then

$$
s_{L}([v]) \stackrel{\text { def }}{=}\left[\left(v^{1}, 0, v^{2}, 0, \ldots\right)\right], s_{R}([v]) \stackrel{\text { def }}{=}\left[\left(0, v^{1}, 0, v^{2}, \ldots\right)\right]
$$

We observe that $s_{L}, s_{R}$ are homotopic to the identity. It follows that, if we define

$$
\tilde{w}:\left(\left[b_{L}, c_{L}\right],\left[b_{R}, c_{R}\right]\right) \mapsto\left[s_{L}\left(b_{L}\right) \oplus s_{R}\left(b_{R}\right), s_{L}\left(c_{L}\right) \oplus s_{R}\left(c_{R}\right)\right]
$$

then

$$
(\tilde{w})^{*} \tilde{E}_{u}=\tilde{L}_{u} \oplus \tilde{p r^{*}} \tilde{L}_{u},(\tilde{w})^{*} \tilde{E}_{v}=\tilde{L}_{v} \oplus \tilde{p r^{*}} \tilde{L}_{v}
$$

It remain to show diagram 17 is commutative. Let $j_{z}: F_{0}(1, \infty) \rightarrow N_{z}$ be defined by $j_{z}:[b, c] \mapsto[z, 0, b, c]$. Then the diagram

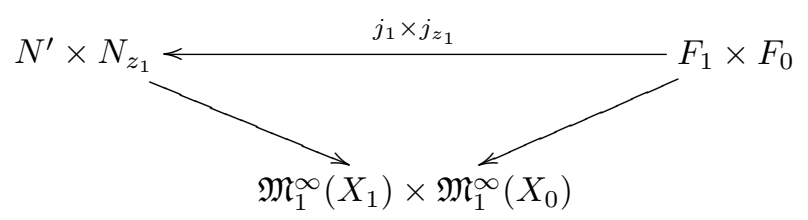

is homotopy commutative. We are left with the diagram

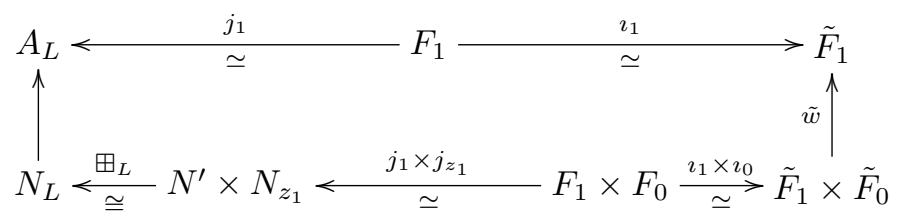

Now define the map $w: F_{1}(1, \infty) \times F_{0}(1, \infty) \rightarrow F_{1}(2, \infty)$ by

$$
w:\left(\left[b_{L}, c_{L}\right],\left[b_{R}, c_{R}\right]\right) \mapsto\left[s_{L}\left(b_{L}\right) \oplus s_{R}\left(b_{R}\right), s_{L}\left(c_{L}\right) \oplus s_{R}\left(c_{R}\right)\right]
$$

Clearly we have the commutative diagram

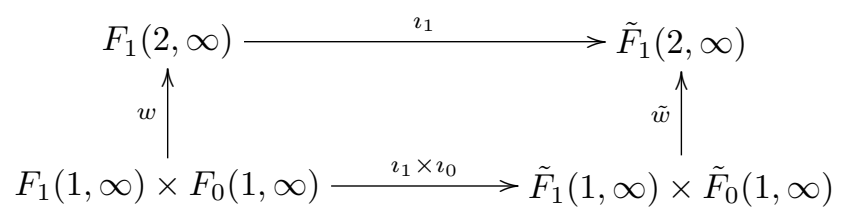


We are thus left with the diagram

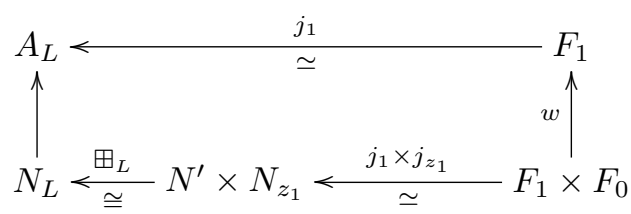

Next we introduce maps

$$
S_{L}([b, c])=\left[s_{L}(b), s_{L}(c)\right], S_{R}([b, c])=\left[s_{R}(b), s_{R}(c)\right]
$$

These maps are homotopic to the identity hence we only have to show the diagram

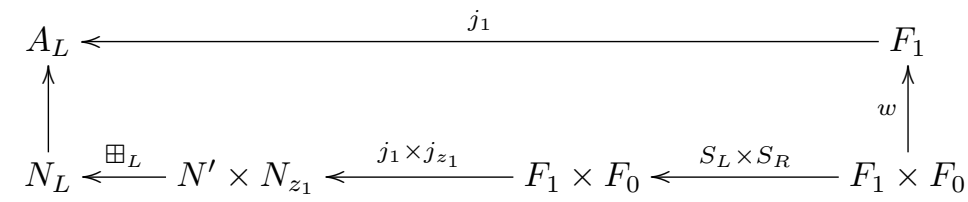

is homotopy commutative. This is an easy direct verification.

(4) We want to show that $\left.E_{0}\right|_{N_{0}} \cong L_{0 L} \oplus L_{0 R}$. Consider the diagram

$$
\begin{gathered}
N_{0} \stackrel{i_{1}}{\longrightarrow} A_{0} \\
\vee^{i_{2}} \stackrel{\downarrow^{i_{3}}}{N_{L} \stackrel{i_{4}}{\longrightarrow} A_{L}}
\end{gathered}
$$

Then $E_{0}=i_{3}^{*} E_{b L}$ so

$$
\left.E_{0}\right|_{N_{0}}=i_{1}^{*} E_{0}=i_{1}^{*} i_{3}^{*} E_{b L}=i_{2}^{*} i_{4}^{*} E_{b L}=L_{0 L} \oplus L_{0 R}
$$

(5) We want to show that $\left.\left.L_{b R}\right|_{N_{L}} \cong L_{c R}\right|_{N_{L}} \cong L_{0 R}$. The result will follow if we show that the following diagram is homotopy commutative:

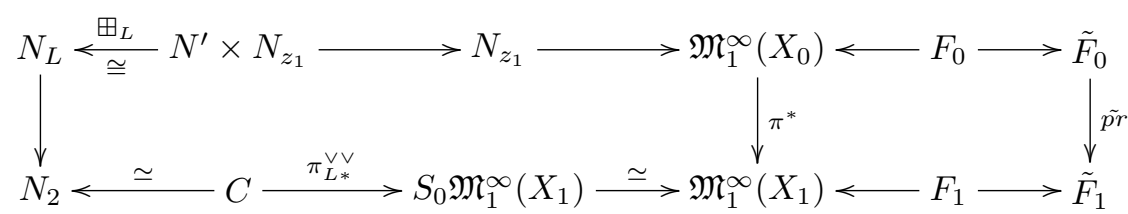

Let

$$
\begin{aligned}
& S_{1 L} N_{2}=\left\{(\mathcal{E}, \phi) \in N_{2} \mid c_{2}\left(\left(\pi_{L *} \mathcal{E}\right)^{\vee \vee}\right)=1\right\} \\
& S_{1} N_{L}=\left\{(\mathcal{E}, \phi) \in N_{L} \mid c_{2}\left(\left(\pi_{L *} \mathcal{E}\right)^{\vee \vee}\right)=1\right\} \\
& S_{0} N^{\prime}=\left\{(\mathcal{E}, \phi) \in N^{\prime} \mid c_{2}\left(\left(\pi_{L *} \mathcal{E}\right)^{\vee \vee}\right)=0\right\}
\end{aligned}
$$


Then the commutativity of (19) follows from the commutativity of

$(20)$

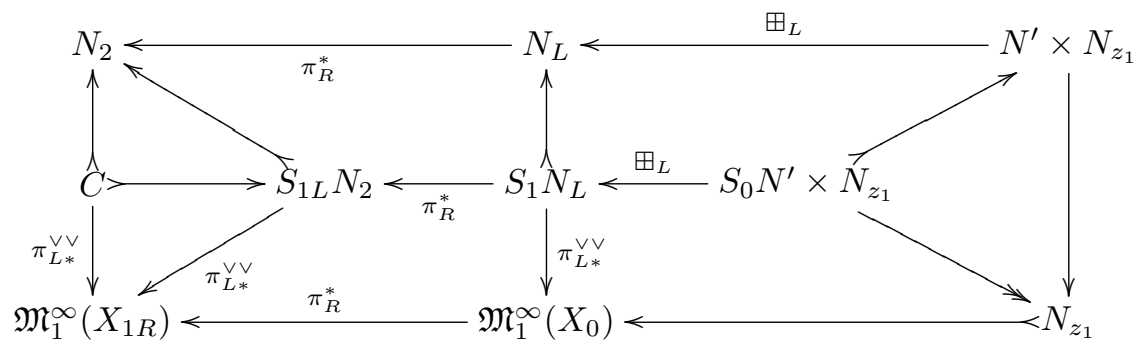

We need to check the image of $\boxplus_{L}: S_{0} N^{\prime} \times N_{z_{1}} \rightarrow N_{L}$ is contained in $S_{1} N_{L}$. Then, analyzing the commutativity of diagram (20) boils down do analyzing the diagram

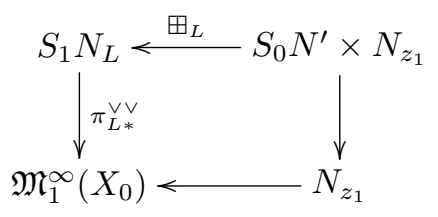

Let $m^{\prime} \in S_{0} N^{\prime} \subset S_{0} \mathfrak{M}_{1}^{\infty}\left(X_{1}\right), m^{\prime}=\left[a_{1}^{\prime}, a_{2}^{\prime}, 0, b^{\prime}, c^{\prime}\right]$. Let $m^{\prime \prime} \in N_{z}$. Then a direct computation shows that $\left(\pi_{L *}\left(m^{\prime} \boxplus_{L} m^{\prime \prime}\right)\right)^{\vee \vee}=m^{\prime \prime}$. This shows that the image of $S_{0} N^{\prime} \times N_{z_{1}}$ under $\boxplus_{L}$ is contained in $S_{1} N_{L}$ and that diagram (21) is commutative.

(6) We want to show that $\left.L_{b L, 2}\right|_{N_{L}} \cong L_{b L, L},\left.L_{c L, 2}\right|_{N_{L}} \cong L_{c L, L}$. This will follow from the commutativity of the diagram

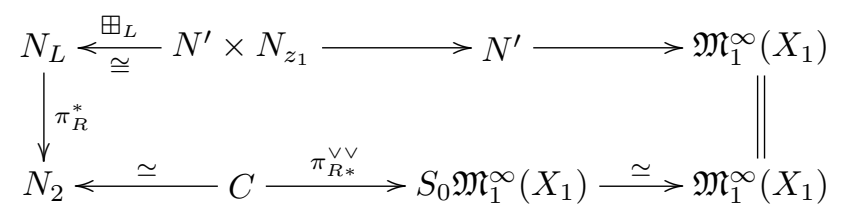

We showed in proposition 4.9 that the map $H_{2}(\cdot, 0)$ is the homotopy inverse of the inclusion $C \rightarrow N_{2}$. Let $\left(m^{\prime}, m^{\prime \prime}\right) \in N^{\prime} \times N_{z}$. Then, by definition of $\mathrm{H}_{2}$,

$\pi_{R *} H_{2}\left(\pi_{R}^{*}\left(m^{\prime} \boxplus_{L} m^{\prime \prime}\right), 0\right)=H_{L}\left(m^{\prime} \boxplus_{L} m^{\prime \prime}, 0\right)=H_{1}\left(m^{\prime}, 0\right) \boxplus H_{z}\left(m^{\prime \prime}, 0\right)$

Hence the diagram

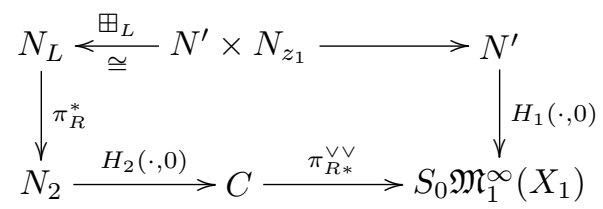

is commutative. From here it follows easily that diagram (22) is commutative.

\section{The Cohomology of $\mathfrak{M}_{2}^{\infty}\left(X_{q}\right)$}

The objective of this section is to prove theorem 1.4. We begin by proving it for the special case $q=2$ : 
Theorem 6.1. There is an exact sequence

$$
\begin{aligned}
0 \rightarrow H^{*}\left(\mathfrak{M}_{2}^{\infty}\left(X_{2}\right)\right) \rightarrow H^{*}\left(A_{L}\right) & \oplus H^{*}\left(A_{R}\right) \oplus H^{*}\left(N_{2}\right) \rightarrow \\
& \rightarrow H^{*}\left(A_{0}\right) \oplus H^{*}\left(N_{L}\right) \oplus H^{*}\left(N_{R}\right) \rightarrow H^{*}\left(N_{0}\right) \rightarrow 0
\end{aligned}
$$

which splits to give an isomorphism

$$
\begin{aligned}
H^{*}\left(\mathfrak{M}_{2}^{\infty}\left(X_{2}\right)\right) \cong \operatorname{Ker}\left(H^{*}\left(A_{L}\right) \oplus H^{*}\left(A_{R}\right) \rightarrow H^{*}\left(A_{0}\right)\right) \oplus \\
\oplus \operatorname{Ker}\left(H^{*}\left(N_{2}\right) \rightarrow H^{*}\left(N_{L}\right) \oplus H^{*}\left(N_{R}\right)\right)
\end{aligned}
$$

Proof. Recall corolary 4.2. We will use this spectral sequence to compute $H_{*}\left(\mathfrak{M}_{2}^{\infty}\left(X_{2}\right)\right)$. Clearly the map $d_{1}: E_{1, n} \rightarrow E_{2, n}$ is surjective hence $E_{2}^{2, n}=0$. Also we notice that $E_{1}^{p, 2 n+1}=0$ for any $p$. It follows that the spectral sequence collapses at the term $E_{2}$. We get then

$$
\begin{gathered}
H^{2 n}\left(\mathfrak{M}_{2}^{\infty}\left(X_{2}\right)\right)=E_{\infty}^{0,2 n}=\operatorname{Ker}\left(d_{1}: E_{1}^{0,2 n} \rightarrow E_{1}^{1,2 n}\right) \\
H^{2 n+1}\left(\mathfrak{M}_{2}^{\infty}\left(X_{2}\right)\right)=E_{\infty}^{1,2 n}=\frac{\operatorname{Ker}\left(d_{1}: E_{1}^{1,2 n} \rightarrow E_{1}^{2,2 n}\right)}{\operatorname{Im}\left(d_{1}: E_{1}^{0,2 n} \rightarrow E_{1}^{1,2 n}\right)}
\end{gathered}
$$

When performing calculations we will use the following sign conventions:

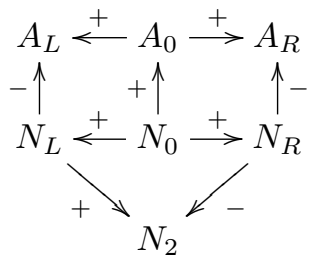

We begin by defining the following generators of the cohomology of $E_{1}^{0,2 n}$ :

$$
\begin{array}{ll}
a_{\Delta L}^{i}=c_{i}\left(E_{c L}\right)-c_{i}\left(E_{b L}\right) & a_{\Delta R}^{i}=c_{i}\left(E_{c R}\right)-c_{i}\left(E_{b R}\right) \\
a_{b L}^{i}=c_{i}\left(E_{b L}\right) & a_{b R}^{i}=c_{i}\left(E_{b R}\right) \\
c_{\Delta L}=c_{1}\left(L_{c L}\right)-c_{1}\left(L_{b L}\right) & c_{\Delta R}=c_{1}\left(L_{c R}\right)-c_{1}\left(L_{b R}\right) \\
c_{b L}=c_{1}\left(L_{b L}\right) & c_{b R}=c_{1}\left(L_{b R}\right)
\end{array}
$$

We do the same for $E_{1}^{1,2 n}$ :

$$
\begin{array}{ll}
n_{\Delta L}=c_{1}\left(L_{c L}\right)-c_{1}\left(L_{b L}\right) & n_{\Delta R}=c_{1}\left(L_{c R}\right)-c_{1}\left(L_{b R}\right) \\
n_{b L}=c_{1}\left(L_{b L}\right) & n_{b R}=c_{1}\left(L_{b R}\right) \\
n_{0 R}=c_{1}\left(L_{0 R}\right) & n_{0 L}=c_{1}\left(L_{0 L}\right) \\
a^{i}=c_{i}\left(E_{0}\right) &
\end{array}
$$

and for $E_{1}^{2, n}$ :

$$
n_{0 R}=c_{1}\left(L_{0 R}\right) \quad n_{0 L}=c_{1}\left(L_{0 L}\right)
$$

Then, from theorem 5.2 it follows that the map $d_{1}: E_{1}^{0,2 n} \rightarrow E_{1}^{1,2 n}$ may be represented by the following diagram, whose entries correspond to those in diagram 
(25):

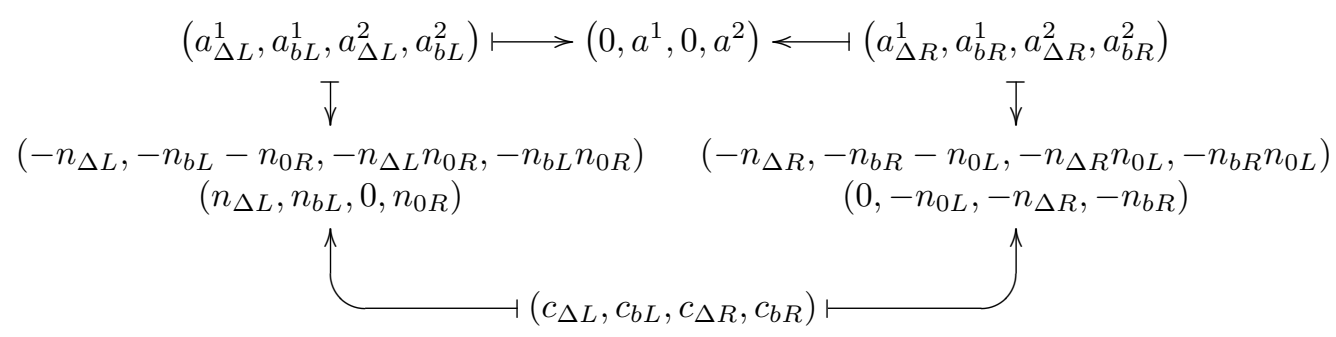

Also the map $d_{1}: E_{1}^{1,2 n} \rightarrow E_{1}^{2,2 n}$ is given by

$$
\begin{aligned}
& \left(a^{1}, a^{2}\right) \mapsto\left(n_{0 L}+n_{0 R}, n_{0 L} n_{0 R}\right) \\
& \left(n_{\Delta L}, n_{b L}, n_{0 R}\right) \mapsto\left(0, n_{0 L}, n_{0 R}\right) \\
& \left(n_{0 L}, n_{\Delta R}, n_{b R}\right) \mapsto\left(n_{0 L}, 0, n_{0 R}\right)
\end{aligned}
$$

Now let

$$
\begin{array}{ll}
K_{A L}=\operatorname{Ker}\left(H^{*}\left(A_{L}\right) \rightarrow H^{*}\left(A_{0}\right)\right) & K_{A R}=\operatorname{Ker}\left(H^{*}\left(A_{R}\right) \rightarrow H^{*}\left(A_{0}\right)\right) \\
K_{N L}=\operatorname{Ker}\left(H^{*}\left(N_{L}\right) \rightarrow H^{*}\left(N_{0}\right)\right) & K_{N R}=\operatorname{Ker}\left(H^{*}\left(N_{R}\right) \rightarrow H^{*}\left(N_{0}\right)\right)
\end{array}
$$

Then

$$
\begin{aligned}
& H^{*}\left(A_{L}\right) \cong \mathbb{Z}\left[a^{1}, a^{2}\right] \oplus K_{A L}, H^{*}\left(A_{R}\right) \cong \mathbb{Z}\left[a^{1}, a^{2}\right] \oplus K_{A R} \\
& H^{*}(C) \cong \mathbb{Z}\left[n_{L}, n_{R}\right] \oplus K_{N L} \oplus K_{N R} \oplus K_{C} \\
& H^{*}\left(N_{L}\right) \cong \mathbb{Z}\left[n_{L}, n_{R}\right] \oplus K_{N L}, H^{*}\left(N_{R}\right) \cong \mathbb{Z}\left[n_{L}, n_{R}\right] \oplus K_{N R}
\end{aligned}
$$

Notice that $K_{C} \subset H^{*}(C)$ is the ideal generated by $c_{\Delta L} c_{\Delta R}$. The restriction of the map $H^{*}\left(A_{L}\right) \rightarrow H^{*}\left(N_{L}\right)$ to $K_{A L}$ induces a map $s_{L}: K_{A L} \rightarrow K_{N L}$. Similarly we have a map $s_{R}: K_{A R} \rightarrow K_{N R}$. Let also $s: \mathbb{Z}\left[a^{1}, a^{2}\right] \rightarrow \mathbb{Z}\left[n_{L}, n_{R}\right]$ be the map induced by the direct sum map $B U(1) \times B U(1) \rightarrow B U(2)$. Then the map $d_{1}: E_{1}^{0,2 n} \rightarrow E_{1}^{1,2 n}$ is given by

$$
\begin{aligned}
& d_{1}\left(a_{L}+k_{A L}, a_{R}+k_{A R}, x+k_{N L}+k_{N R}+k_{C}\right)= \\
& \quad=\left(-s\left(a_{L}\right)-s_{L}\left(k_{A L}\right)+x+k_{N L},-s\left(a_{R}\right)-s_{R}\left(k_{A R}\right)-x-k_{N R}, a_{L}+a_{R}\right)
\end{aligned}
$$

and the map $d_{1}: E_{1}^{1,2 n} \rightarrow E_{1}^{2,2 n}$ is given by

$$
d_{1}\left(x_{L}+k_{N L}, x_{R}+k_{N R}, a\right)=\left(x_{L}+x_{R}+s(a)\right)
$$

Now we can finish the proof:

(1) We prove first that $H^{2 n+1}\left(\mathfrak{M}_{2}^{\infty}\left(X_{2}\right)\right)=0$. We need to show $\operatorname{Ker}\left(d_{1}\right.$ : $\left.E_{1}^{1,2 n} \rightarrow E_{1}^{2,2 n}\right) \subset \operatorname{Im}\left(d_{1}: E_{1}^{0,2 n} \rightarrow E_{1}^{1,2 n}\right)$. Let $\left(x_{L}+k_{N L}, x_{R}+k_{N R}, a\right) \in$ Ker $d_{1}$. Then $x_{L}+x_{R}+s(a)=0$. It follows that

$$
d_{1}\left(a, 0,-x_{R}+k_{L}-k_{R}\right)=\left(x_{L}+k_{L}, x_{R}+k_{R}, a\right)
$$

(2) Now we will show that $H^{2 n} \cong \mathbb{Z}\left[a^{1}, a^{2}\right] \oplus K_{A L} \oplus K_{A R} \oplus K_{C}$ which conpletes the proof. We first define a map $\mathbb{Z}\left[a^{1}, a^{2}\right] \oplus K_{A L} \oplus K_{A R} \oplus K_{C} \rightarrow E_{1}^{0,2 n}$ by

$$
\left(a, k_{A L}, k_{A R}, k_{C}\right) \mapsto\left(a+k_{A L},-a+k_{A R}, s(a)+s_{L}\left(k_{A L}\right)-s_{R}\left(k_{A R}\right)+k_{C}\right)
$$


We want to show this map is injective onto the kernel of $d_{1}$. Injectivity is clear and a direct verification shows the image is contained in the kernel of $d_{1}$. To show surjectivity let

$$
\left(a_{L}+k_{A L}, a_{R}+k_{A R}, x+k_{N L}+k_{N R}+k_{C}\right) \in \operatorname{Ker} d_{1} .
$$

Then $a_{L}=-a_{R}, k_{N L}=s_{L}\left(k_{A L}\right), k_{N R}=-s_{R}\left(k_{A R}\right)$ and $x=s\left(a_{L}\right)=$ $-s\left(a_{R}\right)$. The result follows.

We are ready to prove the general case:

Theorem 6.2. With notations as in theorem 2.1 let

$$
\begin{aligned}
& K_{i}=\operatorname{Ker}\left(H^{*}\left(\pi_{i}^{*} \mathfrak{M}_{2}^{\infty}\left(X_{1}\right)\right) \rightarrow H^{*}\left(\pi_{\emptyset}^{*} \mathfrak{M}_{2}^{\infty}\left(X_{0}\right)\right)\right) \\
& K_{i j}=\operatorname{Ker}\left(H^{*}\left(\pi_{i j}^{*} \mathfrak{M}_{2}^{\infty}\left(X_{2}\right)\right) \rightarrow H^{*}\left(\pi_{i}^{*} \mathfrak{M}_{2}^{\infty}\left(X_{1}\right)\right) \oplus H^{*}\left(\pi_{j}^{*} \mathfrak{M}_{2}^{\infty}\left(X_{1}\right)\right)\right)
\end{aligned}
$$

Then, as modules over $\mathbb{Z}$, we have an isomorphism

$$
H^{*}\left(\mathfrak{M}_{2}^{\infty}\left(X_{q}\right)\right) \cong H^{*}\left(\mathfrak{M}_{2}^{\infty}\left(X_{0}\right)\right) \oplus \bigoplus_{i} K_{i} \oplus \bigoplus_{i<j} K_{i j}
$$

Proof. We divide the proof into two steps:

(1) We will use theorem 2.1 to build a spectral sequence converging to the cohomology of $H^{*}\left(\mathfrak{M}_{2}^{\infty}\left(X_{q}\right)\right.$. Let $\Delta$ be the $q-1$ simplex. Label its vertices by $v_{i}, i=1, \ldots, q$, and the $e_{i j}$ be the middle point of the edge joining $v_{i}$ and $v_{j}$. We define a filtration $\Delta_{0} \subset \Delta_{1} \subset \Delta$ of $\Delta$ where $\Delta_{0}=\bigcup_{i<j} e_{i j}$ and $\Delta_{1}$ is the 1 -skeleton of $\Delta$. Write $\Delta_{1}=\bigcup_{i} \Delta_{1 i}$ where $\Delta_{1 i}$ is the closure of the connected component of $\Delta_{1} \backslash \Delta_{0}$ containing $v_{i}$. Then we define

$M=\frac{\bigcup_{i, j}\left(e_{i j} \times \pi_{i j}^{*} \mathfrak{M}_{2}^{\infty}\left(X_{2}\right)\right) \cup \bigcup_{i}\left(\Delta_{1 i} \times \pi_{i}^{*} \mathfrak{M}_{2}^{\infty}\left(X_{1}\right)\right) \cup\left(\Delta \times \pi_{\emptyset}^{*} \mathfrak{M}_{2}^{\infty}\left(X_{0}\right)\right)}{\sim}$

where $\sim$ is induced by the inclusions $e_{i j} \subset \Delta_{1 i} \subset \Delta$ and $\pi_{\emptyset}^{*} \mathfrak{M}_{2}^{\infty}\left(X_{0}\right) \subset$ $\pi_{i}^{*} \mathfrak{M}_{2}^{\infty}\left(X_{1}\right) \subset \pi_{i j}^{*} \mathfrak{M}_{2}^{\infty}\left(X_{2}\right)$. Then, the arguments in [21] can be applied to show that $M$ is homotopically equivalent to $\mathfrak{M}_{2}^{\infty}\left(X_{q}\right)$. The filtration of $\Delta$ by $\Delta_{0}, \Delta_{1}$ induces a filtration $F_{0} \subset F_{1} \subset F_{2}=M$ of $M$ which leads to a spectral sequence with

$$
\begin{aligned}
& E_{1}^{0, n}=H^{n}\left(F_{0}\right) \cong \bigoplus_{i<j}\left(H^{0}\left(e_{i j}\right) \otimes H^{n}\left(\pi_{i j}^{*} \mathfrak{M}_{2}^{\infty}\left(X_{2}\right)\right)\right) \\
& E_{1}^{1, n}=H^{n}\left(F_{1}, F_{0}\right) \cong \bigoplus_{i}\left(H^{1}\left(\Delta_{1 i}, \partial \Delta_{1 i}\right) \otimes H^{n}\left(\pi_{i}^{*} \mathfrak{M}_{2}^{\infty}\left(X_{1}\right)\right)\right) \\
& E_{1}^{2, n}=H^{n}\left(F_{2}, F_{1}\right) \cong H^{1}\left(F_{1}\right) \otimes H^{n}\left(\mathfrak{M}_{2}^{\infty}\left(X_{0}\right)\right)
\end{aligned}
$$

(2) The $d_{1}$ differential is induced by the inclusions $\pi_{\emptyset}^{*} \mathfrak{M}_{2}^{\infty}\left(X_{0}\right) \rightarrow \pi_{i}^{*} \mathfrak{M}_{2}^{\infty}\left(X_{1}\right) \rightarrow$ $\pi_{i j}^{*} \mathfrak{M}_{2}^{\infty}\left(X_{2}\right)$. We will use the sign conventions $(i<j)$ :

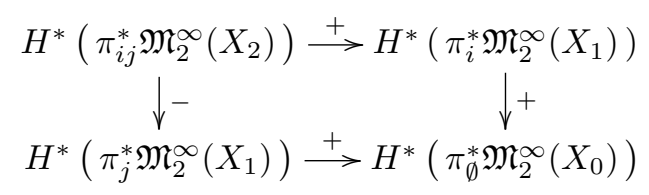


Let

$$
\begin{aligned}
& K_{i}=\operatorname{Ker}\left(H^{*}\left(\pi_{i}^{*} \mathfrak{M}_{2}^{\infty}\left(X_{1}\right)\right) \rightarrow H^{*}\left(\pi_{\emptyset}^{*} \mathfrak{M}_{2}^{\infty}\left(X_{0}\right)\right)\right) \\
& K_{i j}=\operatorname{Ker}\left(H^{*}\left(\pi_{i j}^{*} \mathfrak{M}_{2}^{\infty}\left(X_{2}\right)\right) \rightarrow H^{*}\left(\pi_{i}^{*} \mathfrak{M}_{2}^{\infty}\left(X_{1}\right)\right) \oplus H^{*}\left(\pi_{j}^{*} \mathfrak{M}_{2}^{\infty}\left(X_{1}\right)\right)\right)
\end{aligned}
$$

Then, from theorem 6.1 we have

$$
\begin{aligned}
& H^{*}\left(\pi_{i}^{*} \mathfrak{M}_{2}^{\infty}\left(X_{1}\right)\right) \cong H^{*}\left(\pi_{\emptyset}^{*} \mathfrak{M}_{2}^{\infty}\left(X_{0}\right)\right) \oplus K_{i} \\
& H^{*}\left(\pi_{i j}^{*} \mathfrak{M}_{2}^{\infty}\left(X_{2}\right)\right) \cong H^{*}\left(\pi_{\emptyset}^{*} \mathfrak{M}_{2}^{\infty}\left(X_{0}\right)\right) \oplus K_{i} \oplus K_{j} \oplus K_{i j}
\end{aligned}
$$

Then the sequence of maps $E_{1}^{0, n} \stackrel{d_{1}}{\rightarrow} E_{1}^{1, n} \stackrel{d_{1}}{\rightarrow} E_{1}^{2, n}$ splits into three sequences

$$
\bigoplus_{i<j} H^{0}\left(e_{i j}\right) \otimes K_{i j} \longrightarrow 0 \longrightarrow 0
$$

$\bigoplus_{i<j} H^{0}\left(e_{i j}\right) \otimes\left(K_{i} \oplus K_{j}\right) \longrightarrow \bigoplus_{l} H^{1}\left(\Delta_{1 l}, \partial \Delta_{1 l}\right) \otimes K_{l} \longrightarrow 0$

$$
H^{0}\left(\Delta_{0}\right) \otimes K^{n} \longrightarrow H^{1}\left(\Delta_{1}, \Delta_{0}\right) \otimes K^{n} \longrightarrow H^{1}\left(\Delta_{1}\right) \otimes K^{n}
$$

where $K^{n}$ stands for $H^{n}\left(\mathfrak{M}_{2}^{\infty}\left(X_{0}\right)\right)$. The bottom maps are easily analyzed using the exact sequence

$$
0 \rightarrow H^{0}\left(\Delta_{1}\right) \rightarrow H^{0}\left(\Delta_{0}\right) \rightarrow H^{1}\left(\Delta_{1}, \Delta_{0}\right) \rightarrow H^{1}\left(\Delta_{1}\right) \rightarrow 0
$$

It follows that the map $d_{1}: E_{1}^{1, n} \rightarrow E_{1}^{2, n}$ is surjective. Since $E_{1}^{r, n}=0$ for $r>2$ and $n$ even, this implies the spectral sequence collapses and

$$
\begin{gathered}
H^{n}\left(\mathfrak{M}_{2}^{\infty}\left(X_{q}\right)\right)=\frac{\operatorname{Ker}\left(d_{1}: E_{1}^{1, n} \rightarrow E_{1}^{2, n}\right)}{\operatorname{Im}\left(d_{1}: E_{1}^{0, n} \rightarrow E_{1}^{1, n}\right)} \\
H^{n}\left(\mathfrak{M}_{2}^{\infty}\left(X_{q}\right)\right)=\operatorname{Ker}\left(d_{1}: E_{1}^{0, n} \rightarrow E_{1}^{1, n}\right)
\end{gathered}
$$

Lets look more closely at the map

$$
\bigoplus_{i<j} H^{0}\left(e_{i j}\right)\left(K_{i} \oplus K_{j}\right) \rightarrow \bigoplus_{i} H^{1}\left(\Delta_{1 i}, \partial \Delta_{1 i}\right) \otimes K_{i}
$$

Observe that

$$
\bigoplus_{i<j} H^{0}\left(e_{i j}\right) \otimes\left(K_{i} \oplus K_{j}\right)=\bigoplus_{i} H^{0}\left(\partial \Delta_{1 i}\right) \otimes K_{i}
$$

It follows that the map (29) can be easily analysed using the exact sequence

$$
0 \rightarrow H^{0}\left(\Delta_{1 i}\right) \rightarrow H^{0}\left(\partial \Delta_{1 i}\right) \rightarrow H^{1}\left(\Delta_{1 i}, \partial \Delta_{1 i}\right) \rightarrow 0
$$

We gather together our conclusions:

(a) The top sequence in (28) contributes a term

$$
\bigoplus_{i<j} H^{0}\left(e_{i j}\right) \otimes K_{i j}
$$

to $H^{2 n}\left(\mathfrak{M}_{2}^{\infty}\left(X_{q}\right)\right)$.

(b) The bottom sequence in (28) does not contribute to $H^{2 n+1}\left(\mathfrak{M}_{2}^{\infty}\left(X_{q}\right)\right)$ since it is exact in the middle.

(c) The bottom sequence in (28) contributes a term

$$
H^{0}\left(\Delta_{1}\right) \otimes H^{*}\left(\mathfrak{M}_{2}^{\infty}\left(X_{0}\right)\right)
$$

to $H^{2 n}\left(\mathfrak{M}_{2}^{\infty}\left(X_{q}\right)\right)$. 
(d) The map (29) is surjective hence it does not contribute to $H^{2 n+1}\left(\mathfrak{M}_{2}^{\infty}\left(X_{q}\right)\right)$.

(e) The map (29) contributes a term

$$
\bigoplus_{i} H^{0}\left(\Delta_{1 i}\right) \otimes K_{i}
$$

to $H^{2 n}\left(\mathfrak{M}_{2}^{\infty}\left(X_{q}\right)\right)$.

From (b) and (d) it follows that $H^{2 n+1}\left(\mathfrak{M}_{2}^{\infty}\left(X_{q}\right)\right)=0$ and from (a), (c) and (e) equation (26) follows.

\section{Appendix A. Monads}

In this appendix we will sketch the monad description of the spaces $\mathfrak{M}_{k}^{r}\left(\mathbb{C P}^{2}\right)$ and $\mathfrak{M}_{k}^{r}\left(\tilde{\mathbb{C P}}^{2}\right)$. We follow [12]. See also [3].

Let $L_{\infty} \subset \mathbb{C P}^{2}$ be a rational curve and let $L \subset \tilde{\mathbb{C P}}^{2}$ be the exceptional divisor. Choose sections $x_{1}, x_{2}, x_{3}$ spanning $H^{0}\left(\mathcal{O}\left(L_{\infty}\right)\right)$ and $y_{1}, y_{2}$ spanning $H^{0}\left(\mathcal{O}\left(L_{\infty}-L\right)\right)$ so that $x_{3}$ vanishes on $L_{\infty}$ and $x_{1} y_{1}+x_{2} y_{2}$ spans the kernel of

$$
H^{0}\left(\mathcal{O}\left(L_{\infty}\right)\right) \otimes H^{0}\left(\mathcal{O}\left(L_{\infty}-L\right)\right) \longrightarrow H^{0}\left(\mathcal{O}\left(2 L_{\infty}-L\right)\right)
$$

A.1. The moduli space over $\mathbb{C P}^{2}$. Let $W$ be a $k$-dimensional vector space. Let $\mathcal{R}$ be the space of 4 -tuples $m=\left(a_{1}, a_{2}, b, c\right)$ with $a_{i} \in \operatorname{End}(W), b \in \operatorname{Hom}\left(\mathbb{C}^{r}, W\right)$, $c \in \operatorname{Hom}\left(W, \mathbb{C}^{r}\right)$, obeying the integrability condition $\left[a_{1}, a_{2}\right]+b c=0$. For each $m=\left(a_{1}, a_{2}, b, c\right) \in \mathcal{R}$ we define maps $A_{m}, B_{m}$

$$
W\left(-L_{\infty}\right) \stackrel{A_{m}}{\longrightarrow} W^{\oplus 2} \oplus \mathbb{C}^{n} \stackrel{B_{m}}{\longrightarrow} W\left(L_{\infty}\right)
$$

by

$$
A_{m}=\left[\begin{array}{c}
x_{1}-a_{1} x_{3} \\
x_{2}-a_{2} x_{3} \\
c x_{3}
\end{array}\right], B_{m}=\left[\begin{array}{lll}
-x_{2}+a_{2} x_{3} & x_{1}-a_{1} x_{3} & b x_{3}
\end{array}\right]
$$

Then $B_{m} A_{m}=0$. The assignement $m \mapsto \mathcal{E}_{m}=\operatorname{Ker} B_{m} / \operatorname{Im} A_{m}$ induces a map $f: \mathcal{R} \rightarrow \overline{\mathfrak{M}_{k}^{r}\left(\mathbb{C P}^{2}\right)} . m$ is called non degenerate if $A_{m}, B_{m}$ have maximal rank at every point in $\mathbb{C P}^{2}$.

Theorem A.1. $f$ induces an isomorphism betwen the quotient of the space of non degenerate points in $\mathcal{R}$ by the action of $G l(W)$ :

$$
g \cdot\left(a_{1}, a_{2}, b, c\right)=\left(g^{-1} a_{1} g, g^{-1} a_{2} g, g^{-1} b, c g\right)
$$

and the moduli space $\mathfrak{M}_{k}^{r}\left(\mathbb{C P}^{2}\right)$.

For a proof see [7], proposition 1.

Theorem A.2. The algebraic quotient $\mathcal{R} / G l(W)$ is isomorphic to the DonaldsonUhlenbeck completion of the moduli space of instantons over $S^{4}$.

For a proof see [8], sections 3.3, 3.4, 3.4.4.

We sketch here how the map from $\mathcal{R} / G l(W)$ to the Donaldson-Uhlenbeck completion of the moduli space of instantons is constructed (see [12] for details): Let $m=\left(a_{1}, a_{2}, b, c\right) \in \mathcal{R}$. A subspace $W^{\prime} \subset W$ is called $b$-special with respect to $m$ if

$$
a_{i}\left(W^{\prime}\right) \subset W^{\prime}(i=1,2) \text { and } \operatorname{Im} b \subset W^{\prime} .
$$

A subspace $W^{\prime} \subset W$ is called $c$-special with respect to $m$ if

$$
a_{i}\left(W^{\prime}\right) \subset W^{\prime}(i=1,2) \text { and } W^{\prime} \subset \operatorname{Ker} c .
$$


$m$ is called completely reducible if for every $W^{\prime} \subset W$ which is $b$-special or $c$-special, there is a complement $W^{\prime \prime} \subset W$ which is $c$-special or $b$-special respectively.

Proposition A.3. Let $m=\left(a_{1}, a_{2}, b, c\right) \in \mathcal{R}$.

(1) $m$ is non degenerate if and only if the only b-special subspace is $W$ and the only c-special subspace is 0 ;

(2) For every $m$, the orbit of $m$ under $G l(W)$ contains in its closure a canonical completely reducible orbit and completely reducible orbits have disjoint closures;

(3) If $m$ is completely reducible then, after acting with some $g \in G l(W)$ we can write

$$
a_{i}=\left[\begin{array}{cc}
a_{i}^{r e d} & 0 \\
0 & a_{i}^{\Delta}
\end{array}\right], b=\left[\begin{array}{c}
b^{r e d} \\
0
\end{array}\right], c=\left[\begin{array}{cc}
c^{r e d} & 0
\end{array}\right]
$$

where $\left(a_{1}^{\text {red }}, a_{2}^{\text {red }}, b^{\text {red }}, c^{\text {red }}\right)$ is non-degenerate and the matrices $a_{1}^{\Delta}, a_{2}^{\Delta}$ can be simultaneously diagonalized. Such a configuration is equivalent to the following data:

- An irreducible integrable configuration $\left(a_{1}^{\text {red }}, a_{2}^{\text {red }}, b^{\text {red }}, c^{\text {red }}\right)$ corresponding to a bundle with $c_{2}=l \leq k$;

- $k-l$ points in $\mathbb{C}^{2}=\mathbb{C P}^{2} \backslash L_{\infty}$ given by the eigenvalue pairs of $a_{1}^{\Delta}, a_{2}^{\Delta}$ This is precisely the Donaldson-Uhlenbeck completion.

A.2. The moduli space over $\tilde{\mathbb{C P}}^{2}$. Let $\tilde{\mathcal{R}}$ be the space of 5 -tuples $\tilde{m}=\left(a_{1}, a_{2}, d, b, c\right)$ where $a_{i} \in \operatorname{Hom}(W, V), d \in \operatorname{Hom}(V, W), b \in \operatorname{Hom}\left(\mathbb{C}^{r}, V\right), c \in \operatorname{Hom}\left(W, \mathbb{C}^{r}\right)$, such that $a_{1}(W)+a_{2}(W)+b\left(\mathbb{C}^{r}\right)=V$, obeying the integrability condition $a_{1} d a_{2}-$ $a_{2} d a_{1}+b c=0$. For each $\tilde{m}=\left(a_{1}, a_{2}, d, b, c\right) \in \tilde{\mathcal{R}}$ we define maps $A_{\tilde{m}}, B_{\tilde{m}}$

$$
W\left(-L_{\infty}\right) \oplus V\left(L-L_{\infty}\right) \stackrel{A_{\tilde{m}}}{\longrightarrow}(V \oplus W)^{\oplus 2} \oplus \mathbb{C}^{n} \stackrel{B_{\tilde{m}}}{\longrightarrow} V\left(L_{\infty}\right) \oplus W\left(L_{\infty}-L\right)
$$

by

$$
A_{\tilde{m}}=\left[\begin{array}{cc}
a_{1} x_{3} & -y_{2} \\
x_{1}-d a_{1} x_{3} & 0 \\
a_{2} x_{3} & y_{1} \\
x_{2}-d a_{2} x_{3} & 0 \\
c x_{3} & 0
\end{array}\right], B_{\tilde{m}}=\left[\begin{array}{ccccc}
x_{2} & a_{2} x_{3} & -x_{1} & -a_{1} x_{3} & b x_{3} \\
d y_{1} & y_{1} & d y_{2} & y_{2} & 0
\end{array}\right]
$$

Then $B_{\tilde{m}} A_{\tilde{m}}=0$. The assignement $\tilde{m} \mapsto \mathcal{E}_{\tilde{m}}=\operatorname{Ker} B_{\tilde{m}} / \operatorname{Im} A_{\tilde{m}}$ induces a map $\tilde{f}: \tilde{\mathcal{R}} \rightarrow \overline{\mathfrak{M}_{k}^{r}\left(\tilde{\mathbb{C P}}^{2}\right)}$. A point $\tilde{m} \in \tilde{\mathcal{R}}$ is called non-degenerate if $A_{\tilde{m}}$ and $B_{\tilde{m}}$ have maximal rank at every point in $\tilde{\mathbb{C P}}^{2}$.

Theorem A.4. The map $\tilde{f}$ induces an isomorphism betwen the quotient of the space of non degenerate points in $\tilde{\mathcal{R}}$ by the action of $G l(V) \times G l(W)$ :

$$
\left(g_{0}, g_{1}\right) \cdot\left(a_{1}, a_{2}, b, c, d\right)=\left(g_{0}^{-1} a_{1} g_{1}, g_{0}^{-1} a_{2} g_{1}, g_{0}^{-1} b, c g_{1}, g_{1}^{-1} d g_{0}\right)
$$

and the moduli space $\mathfrak{M}_{k}^{r}\left(\tilde{\mathbb{C P}}^{2}\right)$.

See [12] for a proof.

Consider the algebraic quotient $\tilde{\mathcal{R}} / G l(V) \times G l(W)$. This space is a completion of the moduli space $\mathfrak{M}_{k}^{r}\left(\widetilde{\mathbb{C P}}^{2}\right)$. We proceed to give an interpretation of the points in this completion in terms of the Donaldson-Uhlenbeck completion. See [12] for 
details. Let $\tilde{m}=\left(a_{1}, a_{2}, d, b, c\right)$. Let $V^{\prime} \subset V$ and $W^{\prime} \subset W$ and assume $\operatorname{dim} V^{\prime}=$ $\operatorname{dim} W^{\prime}$. The pair $\left(V^{\prime}, W^{\prime}\right)$ is called $b$-special with respect to $\tilde{m}$ if

$$
a_{i}\left(W^{\prime}\right) \subset V^{\prime}(i=1,2), d\left(V^{\prime}\right) \subset W^{\prime} \text { and } \operatorname{Im} b \subset V^{\prime}
$$

The pair $\left(V^{\prime}, W^{\prime}\right)$ is called $c$-special with respect to $\tilde{m}$ if

$$
a_{i}\left(W^{\prime}\right) \subset V^{\prime}(i=1,2), d\left(V^{\prime}\right) \subset W^{\prime} \text { and } W^{\prime} \subset \operatorname{Ker} c
$$

$\tilde{m}$ is called completely reducible if for every pair $\left(V^{\prime}, W^{\prime}\right)$ which is either $b$-special or $c$-special, there are complements $V^{\prime \prime}, W^{\prime \prime}$ to $V^{\prime}$ and $W^{\prime}$ such that the pair $\left(V^{\prime \prime}, W^{\prime \prime}\right)$ is $c$-special or $b$-special respectively.

Proposition A.5. Let $\tilde{m}=\left(a_{1}, a_{2}, d, b, c\right) \in \tilde{R}$.

(1) $\tilde{m}$ is non-degenerate if and only if the only b-special pair is $(V, W)$ and the only c-special pair is $(0,0)$;

(2) For every $\tilde{m}$, the orbit of $\tilde{m}$ under $G l(V) \times G l(W)$ contains in its closure a canonical completely reducible orbit and completely reducible orbits have disjoint closures;

(3) If $\tilde{m}$ is completely reducible then, after acting with some $\left(g_{0}, g_{1}\right) \in G l(V) \times$ $G l(W)$, we can write

$$
a_{i}=\left[\begin{array}{cc}
a_{i}^{\text {red }} & 0 \\
0 & a_{i}^{\Delta}
\end{array}\right], d=\left[\begin{array}{cc}
d^{r e d} & 0 \\
0 & d^{\Delta}
\end{array}\right] b=\left[\begin{array}{c}
b^{r e d} \\
0
\end{array}\right], c=\left[\begin{array}{ll}
c^{\text {red }} & 0
\end{array}\right]
$$

where $\left(a_{1}^{\text {red }}, a_{2}^{\text {red }}, d^{\text {red }}, b^{\text {red }}, c^{\text {red }}\right)$ is non-degenerate effective and integrable and the matrices $a_{1}^{\Delta}, a_{2}^{\Delta}, d^{\Delta}$ can be simultaneously diagonalized. Such a configuration is equivalent to the following data:

- An irreducible configuration $\left(a_{1}^{\text {red }}, a_{2}^{\text {red }}, d, b^{\text {red }}, c^{\text {red }}\right)$ associated to a bundle with $c_{2}=l \leq k$;

- $k-l$ points in the blow up $\widetilde{\mathbb{C}^{2}}$ of $\mathbb{C}^{2}$ at the origin. This points are determined as follows: $a_{1}^{\Delta}, a_{2}^{\Delta}, d^{\Delta}$ determine $k-l$ unique points $\left(\lambda_{1}^{r}, \lambda_{2}^{r}\right),\left[\mu_{1}^{r}, \mu_{2}^{r}\right] \in \widetilde{\mathbb{C}^{2}}$ corresponding to vectors $v_{1}, \ldots, v_{k-l}$ such that $d a_{i} v^{r}=\lambda_{i}^{r} v^{r}\left(\lambda_{1}, \lambda_{2}\right.$ are the eigenvalue pairs of $\left.d a_{1}, d a_{2}\right)$ and $\left(\mu_{1}^{r} a_{1}+\right.$ $\left.\mu_{2}^{r} a_{2}\right) v^{r}=0$.

A.3. Direct image. In this section we gather some results concerning the direct image map $\pi_{*}$ induced by the blowup map $\pi: \widetilde{C P}^{2} \rightarrow \mathbb{C P}^{2}$.

Proposition A.6. Let $\pi_{\#}: \tilde{\mathcal{R}} \rightarrow \mathcal{R}$ be given by $\pi_{\#}\left(a_{1}, a_{2}, d, b, c\right)=\left(d a_{1}, d a_{2}, d b, c\right)$. Let $\tilde{m} \in \tilde{\mathcal{R}}, m=\pi_{\#} \tilde{m}$. Then $\left.\mathcal{E}_{\tilde{m}}\right|_{\tilde{C P}^{2} \backslash L}$ is isomorphic to $\left.\mathcal{E}_{m}\right|_{\mathbb{C P}^{2} \backslash[0,0,1]}$.

For the proof see [19], proposition 5.6.

Proposition A.7. Let $S_{0} \mathfrak{M}_{1}^{r}\left(\tilde{\mathbb{C P}}^{2}\right)=\left\{(\mathcal{E}, \phi) \in \mathfrak{M}_{1}^{r}\left(\mathbb{C P}^{2}\right):\left(\pi_{*} \mathcal{E}\right)^{\vee \vee}=\mathcal{O}_{\mathbb{C P}^{2}}^{r}\right\}$. Then

(1) $m \in S_{0} \mathfrak{M}_{1}^{r}\left(\mathbb{C P}^{2}\right)$ if and only if $m$ is of the form $\left(a_{1}, a_{2}, 0, b, c\right)$.

(2) The inclusion $S_{0} \mathfrak{M}_{1}^{r} \rightarrow \mathfrak{M}_{1}^{r}$ is a homotopy equivalence.

Proof. First we observe that $\mathfrak{M}_{1}^{r}\left(\tilde{\mathbb{C P}}^{2}\right)=S_{0} \mathfrak{M}_{1}^{r}\left(\tilde{\mathbb{C P}}^{2}\right) \cup \pi_{\emptyset}^{*} \mathfrak{M}_{1}^{r}\left(\mathbb{C P}^{2}\right)$. Now $m \in$ $\pi_{\emptyset}^{*} \mathfrak{M}_{1}^{r}\left(\mathbb{C P}^{2}\right)$ if and only if $d$ is an isomorphism (see [12]). The first statement follows. The second statement follows easily from the first: just consider the homotopy $\left(a_{1}, a_{2}, d, b, c\right) \mapsto\left(a_{1}, a_{2}, t d, b, c\right)$. 
Proposition A.8. Let $x=\left[x_{1}, x_{2}, 1\right] \in \mathbb{C P}^{2}$ and let $\pi_{x}: \tilde{\mathbb{C P}}^{2} \rightarrow \mathbb{C P}^{2}$ be the blow up at $x$. Then the map $\pi_{x}^{*}: \mathfrak{M}_{k}^{r}\left(\mathbb{C P}^{2}\right) \rightarrow \mathfrak{M}_{k}^{r}\left(\tilde{\mathbb{C P}}^{2}\right)$ is given by

$$
\left[a_{1}, a_{2}, b, c\right] \mapsto\left[a_{1}-x_{1} \mathbb{1}, a_{2}-x_{2} \mathbb{1}, \mathbb{1}, b, c\right]
$$

Proof. For $x=[0,0,1]$ see [3]. For the general case consider the translation $\left[w_{1}, w_{2}, w_{3}\right] \mapsto\left[w_{1}-x_{1} w_{3}, w_{2}-x_{2} w_{3}, w_{3}\right]$. This induces a map $\tau: \mathfrak{M}_{k}^{r}\left(X_{0}\right) \rightarrow$ $\mathfrak{M}_{k}^{r}\left(X_{0}\right)$ given by

$$
\left[a_{1}, a_{2}, b, c\right] \mapsto\left[a_{1}-x_{1} \mathbb{1}, a_{2}-x_{2} \mathbb{1}, b, c\right]
$$

The result follows.

Aknowledgements. The author would like to thank professor Ralph Cohen for several fruitful discussions.

\section{REFERENCES}

1. M. F. Atiyah, N. J. Hitchin, V. G. Drinfel'd, and Yu. I. Manin, Construction of instantons, Phys. Lett. A 65 (1978), no. 3, 185-187. MR 82g:81049

2. Jim Bryan and Marc Sanders, The rank stable topology of instantons of $\overline{\mathbf{c p}}^{2}$, Proc. Amer. Math. Soc. 125 (1997), no. 12, 3763-3768. MR 98b:58028

3. _ Instantons on $S^{4}$ and $\overline{\mathbf{C P}}^{2}$, rank stabilization, and Bott periodicity, Topology 39 (2000), no. 2, 331-352. MR 2000k:58011

4. N. P. Buchdahl, Instantons on $\mathbf{c p}_{2}$, J. Differential Geom. 24 (1986), no. 1, 19-52. MR 88b:32066

5. __ Instantons on n $\mathbf{c p}_{2}$, J. Differential Geom. 37 (1993), no. 3, 669-687. MR 94e:53025

6. Nicholas P. Buchdahl, Monads and bundles on rational surfaces, to appear in Rocky Mountain Journal, 2002.

7. S. K. Donaldson, Instantons and geometric invariant theory, Comm. Math. Phys. 93 (1984), no. 4, 453-460. MR 86m:32043

8. S. K. Donaldson and P. B. Kronheimer, The geometry of four-manifolds, The Clarendon Press Oxford University Press, New York, 1990, Oxford Science Publications. MR 92a:57036

9. Elizabeth Gasparim, Holomorphic bundles on $2(-k)$ are algebraic, Comm. Algebra 25 (1997), no. 9, 3001-3009. MR 98f:14037

10. Robin Hartshorne, Algebraic geometry, Springer-Verlag, New York, 1977, Graduate Texts in Mathematics, No. 52. MR 57 \#3116

11. D. Huybrechts and M. Lehn, Stable pairs on curves and surfaces, J. Algebraic Geom. 4 (1995), no. 1, 67-104. MR 95i:14015

12. A. D. King, Instantons and holomorphic bundles on the blown-up plane, Ph.D. thesis, Worcester College, Oxford, 1989.

13. Frances Kirwan, Geometric invariant theory and the Atiyah-Jones conjecture, The Sophus Lie Memorial Conference (Oslo, 1992), Scand. Univ. Press, Oslo, 1994, pp. 161-186. MR 98e:58036

14. Herbert Kurke and Andreas Matuschke, On the structure of moduli spaces of framed vector bundles on rational and ruled surfaces, Algebraic geometry: Hirzebruch 70 (Warsaw, 1998), Amer. Math. Soc., Providence, RI, 1999, pp. 239-271. MR 2000i:14062

15. Manfred Lehn, Modulräume gerahmter Vektorbündel, Universität Bonn Mathematisches Institut, Bonn, 1993, Dissertation, Rheinische Friedrich-Wilhelms-Universität Bonn, Bonn, 1992. MR 95g:14013

16. Martin Lübke, The analytic moduli space of framed vector bundles, J. Reine Angew. Math. 441 (1993), 45-59. MR 94k:32030

17. Andreas Matuschke, On framed instanton bundles and their deformations, Math. Nachr. 211 (2000), 109-126. MR 1743485

18. Marc Sanders, Classifying spaces and Dirac operators coupled to instantons, Trans. Amer. Math. Soc. 347 (1995), no. 10, 4037-4072. MR 96m:58030

19. João P. Santos, Blowups of surfaces and moduli of holomorphic vector bundles, arXiv:math.AG/0212176, 2002. 
20. Topology of moduli spaces of rank stable instantons and holomorphic bundles, Ph.D. thesis, Stanford University, 2002.

21. Graeme Segal, Classifying spaces and spectral sequences, Inst. Hautes Études Sci. Publ. Math. (1968), no. 34, 105-112. MR 38 \#718

Instituto Superior TÉCNICO

E-mail address: jsantos@math.ist.utl.pt 